\title{
WINGS: a WIde-field nearby Galaxy-cluster survey
}

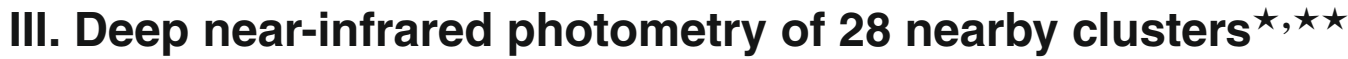

\author{
T. Valentinuzzi ${ }^{1}$, D. Woods ${ }^{2,3}$, G. Fasano ${ }^{4}$, M. Riello ${ }^{5}$, M. D'Onofrio ${ }^{1}$, J. Varela ${ }^{4}$, D. Bettoni ${ }^{4}$, A. Cava ${ }^{4,6}$, \\ W. J. Couch ${ }^{7}$, A. Dressler ${ }^{8}$, J. Fritz ${ }^{4}$, M. Moles ${ }^{9}$, A. Omizzolo ${ }^{10,4}$, B. M. Poggianti ${ }^{4}$, and P. Kjærgaard ${ }^{11}$ \\ 1 Astronomy Department, University of Padova, Vicolo Osservatorio 3, 35122 Padova, Italy \\ e-mail: tiziano.valentinuzzi@unipd.it \\ 2 School of Physics, University of New South Wales, Sydney 2052, NSW, Australia \\ 3 Dept. of Physics \& Astronomy, University of British Columbia, 6224 Agricultural Road, Vancouver, V6T 1Z1, BC, Canada \\ 4 INAF - Padova Astronomical Observatory, Vicolo Osservatorio 5, 35122 Padova, Italy \\ 5 Institute of Astronomy, Madingley Rd., Cambridge CB3 0HA, UK \\ 6 Instituto de Astrofisica de Canarias, via Lactea s.n., 38205 La Laguna, Tenerife, Spain \\ 7 Centre for Astrophysics \& Supercomputing, Swinburne University, Hawthorn 3122, VIC, Australia \\ 8 Observatories of the Carnegie Institution of Washington, Pasadena, CA 91101, USA \\ 9 Instituto de Astrofísica de Andalucía (C.S.I.C.) Apartado 3004, 18080 Granada, Spain \\ ${ }^{10}$ Specola Vaticana, 00120 Stato Citta' del Vaticano, Vatican \\ 11 The Niels Bohr Institute, Juliane Maries Vej 30, 2100 Copenhagen, Denmark
}

Received 29 September 2008 / Accepted 20 February 2009

\begin{abstract}
Context. This is the third paper in a series devoted to the WIde-field Nearby Galaxy-cluster Survey (WINGS). WINGS is a long-term project aimed at gathering wide-field, multiband imaging and spectroscopy of galaxies in a complete sample of $77 \mathrm{X}$-ray selected, nearby clusters $(0.04<z<0.07)$ located far from the galactic plane $\left(|b| \geq 20^{\circ}\right)$. The main goal of this project is to establish a local reference sample for evolutionary studies of galaxies and galaxy clusters.

Aims. This paper presents the near-infrared $(J, K)$ photometric catalogs of 28 clusters of the WINGS sample and describes the procedures followed to construct them.

Methods. The raw data has been reduced at CASU and special care has been devoted to the final coadding, drizzling technique, astrometric solution, and magnitude calibration for the WFCAM pipeline-processed data. We constructed the photometric catalogs based on the final calibrated, coadded mosaics $\left(\approx 0.79 \mathrm{deg}^{2}\right)$ in $J(19$ clusters) and $K(27$ clusters $)$ bands. A customized interactive pipeline was used to clean the catalogs and to make mock images for photometric errors and completeness estimates.

Results. We provide deep near-infrared photometric catalogs $(90 \%$ complete in detection rate at total magnitudes $J \approx 20.5, K \approx 19.4$, and in classification rate at $J \approx 19.5$ and $K \approx 18.5$ ), giving positions, geometrical parameters, total and aperture magnitudes for all detected sources. For each field we classify the detected sources as stars, galaxies, and objects of "unknown" nature.
\end{abstract}

Key words. surveys - galaxies: clusters: general - catalogs

\section{Introduction}

Clusters of galaxies are important for studies of galaxy formation and evolution, because they contain a volume-limited population of galaxies observed at the same cosmic epoch. Galaxies in clusters are known to follow tight color-magnitude relations, which appear to be universal and to have very small intrinsic scatter to the highest redshifts yet observed (see, amongst others, Blakeslee et al. 2003; Holden et al. 2004; Mei et al. 2006; De Lucia et al. 2007). Together with the conventional interpretation of the color-magnitude relation as a mass-metallicity correlation (i.e., Trager et al. 2000), this implies that the majority of the stellar populations in early-type cluster galaxies were formed

* Based on observations taken at the United Kingdom Infra-Red Telescope, operated by the Joint Astronomy Centre on behalf of the Science and Technology Facilities Council of the UK.

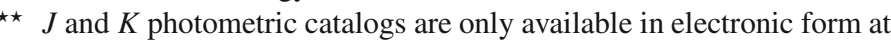
the CDS via anonymous ftp to

cdsarc.u-strasbg.fr $(130.79 .128 .5)$ or via

http://cdsweb.u-strasbg.fr/cgi-bin/qcat?J/A+A/501/851 via rapid dissipative starbursts at $z>2$. Fundamental-plane studies of high-redshift cluster galaxies also support this conclusion, at least for the more massive objects (i.e., van Dokkum \& Stanford 2003; Holden et al. 2005), although the low mass galaxies seem to have undergone more extended star formation histories (i.e., Poggianti et al. 2001).

Theoretically, the existence of such massive and old galaxies at high redshift should represent a strong challenge to models where galaxies are assembled hierarchically, from a sequence of major mergers at progressively lower redshifts. It is not possible, however, to exclude by spectrophotometry alone, these galaxies being assembled from sub-units whose star formation has already ceased, but which are not accreted until later (the so-called "dry" mergers). This is assumed to be the main channel by which spheroids grow at $z<1$ in the hierarchical scenario.

On the other hand, if galaxies are formed via mergers, we should observe a steady decrease in the mean stellar mass in galaxies as we go to earlier cosmic times and the most massive members of the merger tree would branch into even smaller 
Table 1. Summary of the observations, taken with WFCAM on UKIRT, discussed in this paper.

\begin{tabular}{|c|c|c|c|c|c|}
\hline Semester & PATT Ref. & Alloc. & Completion & Observation dates & Comments \\
\hline $2005 \mathrm{~A}$ & $\mathrm{U} / 05 \mathrm{~A} / 2$ & $40 \mathrm{~h}$ & $67 \%$ & $\begin{array}{c}\text { Apr. 29-May } 4 \\
\text { May } 14-15(\mathrm{Pq}) \\
\text { May } 28-29(\mathrm{Pq}), 2005\end{array}$ & $\begin{array}{l}\text { Compromised by poor } \\
\text { weather, seeing and } \\
\text { camera misalignment. }\end{array}$ \\
\hline 2005B & $\mathrm{U} / 05 \mathrm{~B} / 12$ & $40 \mathrm{~h}$ & $18 \%$ & $\begin{array}{c}\text { Sep. } 30 \\
\text { Nov. } 27,2005 \\
\text { Jan. 6, } 2006(\text { all Pq) }\end{array}$ & $\begin{array}{l}\text { Severely } \\
\text { impacted by } \\
\text { poor weather. }\end{array}$ \\
\hline 2006A & $\mathrm{U} / 06 \mathrm{~A} / 12$ & $10 \mathrm{~h}$ & $100 \%$ & $\begin{array}{c}\text { May } 20 \& 26(\mathrm{Pq}) \\
\text { June } 2(\mathrm{Pq}) \\
\text { June } 21 \& 23,2006\end{array}$ & $\begin{array}{l}\text { Good } \\
\text { observing } \\
\text { conditions. }\end{array}$ \\
\hline 2006B & $\mathrm{U} / 06 \mathrm{~B} / 12$ & $14 \mathrm{~h}$ & $65 \%$ & $\begin{array}{c}\text { Dec. 14, } 2006 \\
\text { March 20, 28, } 31 \\
\text { April 3-5, 10-11, } 2007 \text { (all Pq) }\end{array}$ & Average conditions. \\
\hline
\end{tabular}

Note "(Pq)" refers to observations taken in the PATT queue, and not by a WINGS project observer.

twigs (De Lucia et al. 2006). While it is generally difficult to measure galaxy masses, the $K$-band luminosity function is believed to provide an adequate surrogate (Kauffmann \& Charlot 1998). Indeed, it is known that the rest frame $\mathrm{H}$ or $K$ luminosity of galaxies is seen to correlate well with stellar and even dynamical mass for local and high-redshift galaxies (see, i.e., Kodama \& Bower 2003).

To reach higher and higher redshifts it is surely necessary to adequately constrain galaxy formation models; on the other hand, precise knowledge of the properties of clusters and cluster galaxies in the local Universe is necessary, as a benchmark for higher redshift studies. The WIde-field Nearby Galaxy-clusters Survey (Fasano et al. 2006, hereafter Paper I) is an answer to this need. WINGS ${ }^{1}$ is a long-term multiwavelength survey specially designed to provide the first robust characterization of both the photometric and spectroscopic properties of galaxies in nearby clusters, as well as determine the variations in these properties.

The survey core, based on optical $B, V$ imaging of 77 nearby $(\langle z\rangle \approx 0.05)$ galaxy-clusters (see, Varela et al. 2009, hereafter Paper II), has been complemented by several ancillary projects: (i) a spectroscopic follow up of a subsample of 51 clusters, obtained with the spectrographs WYFFOS@WHT and 2dF@AAT; (ii) near-infrared $(J, K)$ imaging of a subsample of 28 clusters obtained with WFCAM@UKIRT, presented here; (iii) $U$ broadand $\mathrm{H}_{\alpha}$ narrow-band imaging of subsamples of WINGS clusters, obtained with wide-field cameras at different telescopes (INT, LBT, Bok). The observations and data reduction for the first two follow-up projects have been completed (Cava et al. 2009; Fritz et al. 2009, in preparation; this paper), while the observations for the $\mathrm{H}_{\alpha}$ and $U$-band surveys are still ongoing.

The near infrared section of the WINGS survey (WINGSNIR) is a collection of $\approx 0.79 \mathrm{deg}^{2}$ mosaics in $(J, K)$ bands of 28 nearby clusters (17 clusters have been observed in both bands).

WINGS-NIR is by far the largest survey of nearby galaxy clusters as far as the area coverage is concerned. In fact, in this redshift range, only individual clusters or small cluster samples have been studied in the literature up to now (e.g., Pahre 1999; Gavazzi et al. 1990; De Propris et al. 2003). The WINGS survey of near-infrared data consists of nearly one million detected sources, with 150000 and 500000 well classified stars and galaxies, respectively.

In Sect. 2 of this paper we describe observations and data reduction techniques, including a brief presentation of both the instrumentation and the software pipeline used to create the final

\footnotetext{
${ }^{1}$ Please refer to WINGS Website for updated details on the survey and its products, http://web. oapd.inaf.it/wings
}

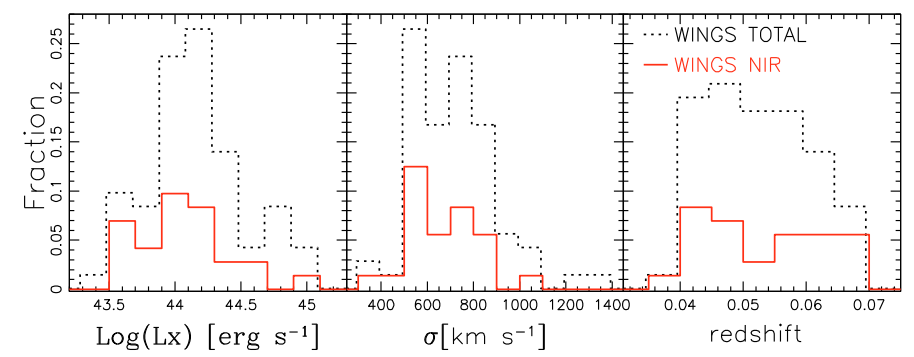

Fig. 1. Distribution of WINGS-NIR clusters (red full line) compared with the distribution of all the WINGS survey clusters (black dotted line). The dotted diagram is slightly shifted for ease of view.

coadded mosaics. Section 3 presents the step-by-step procedure of the catalog production pipeline, including an extensive discussion about star/galaxy classification and interactive cleaning of the catalogs. Section 4 deals with data quality assessment and overall properties of the catalogs, like completeness, astrometric and photometric accuracy and precision. In the last section we present a brief summary of the main features of WINGS-NIR survey and catalogs.

Throughout this paper we will use a cosmological model with parameters: $H_{0}=70 \mathrm{~km} \mathrm{~s}^{-1} \mathrm{Mpc}^{-1}, \Omega_{\mathrm{M}}=0.3$ and $\Omega_{\Lambda}=0.7$.

\section{Observations and data reduction}

The near-infrared data have been collected at the UKIRT telescope using the WFCAM instrument (see, Casali et al. 2007) during four observing semesters, from April 2005 to April 2007 (see Table 1). The images where obtained in the $J$ and $K$ broadbands of the Mauna Kea photometric system ${ }^{2}$ (Tokunaga et al. 2002). The original plan of the WINGS-NIR survey was to image at least all the WINGS clusters with spectroscopy and visible imaging by UKIRT: unfortunately bad weather (see Table 1) limited the sample to only 28 clusters. However, as it can be seen in Fig. 1 the NIR subsample has good coverage of X-ray luminosities and redshifts when compared with the total WINGS sample, while it is slightly biased towards the low cluster velocity dispersion regime.

WFCAM is an assembly of four Rockwell Hawaii-II $2048 \times$ $204818 \mu \mathrm{m}$-pixel array detectors with 0.4 pixel size and a total field of view of $0.21 \mathrm{deg}^{2}$. The four detectors are spaced at

\footnotetext{
${ }^{2}$ Note that WFCAM is equipped with a broad $K$-band filter, not with the short-band $K_{\mathrm{s}}$.
} 


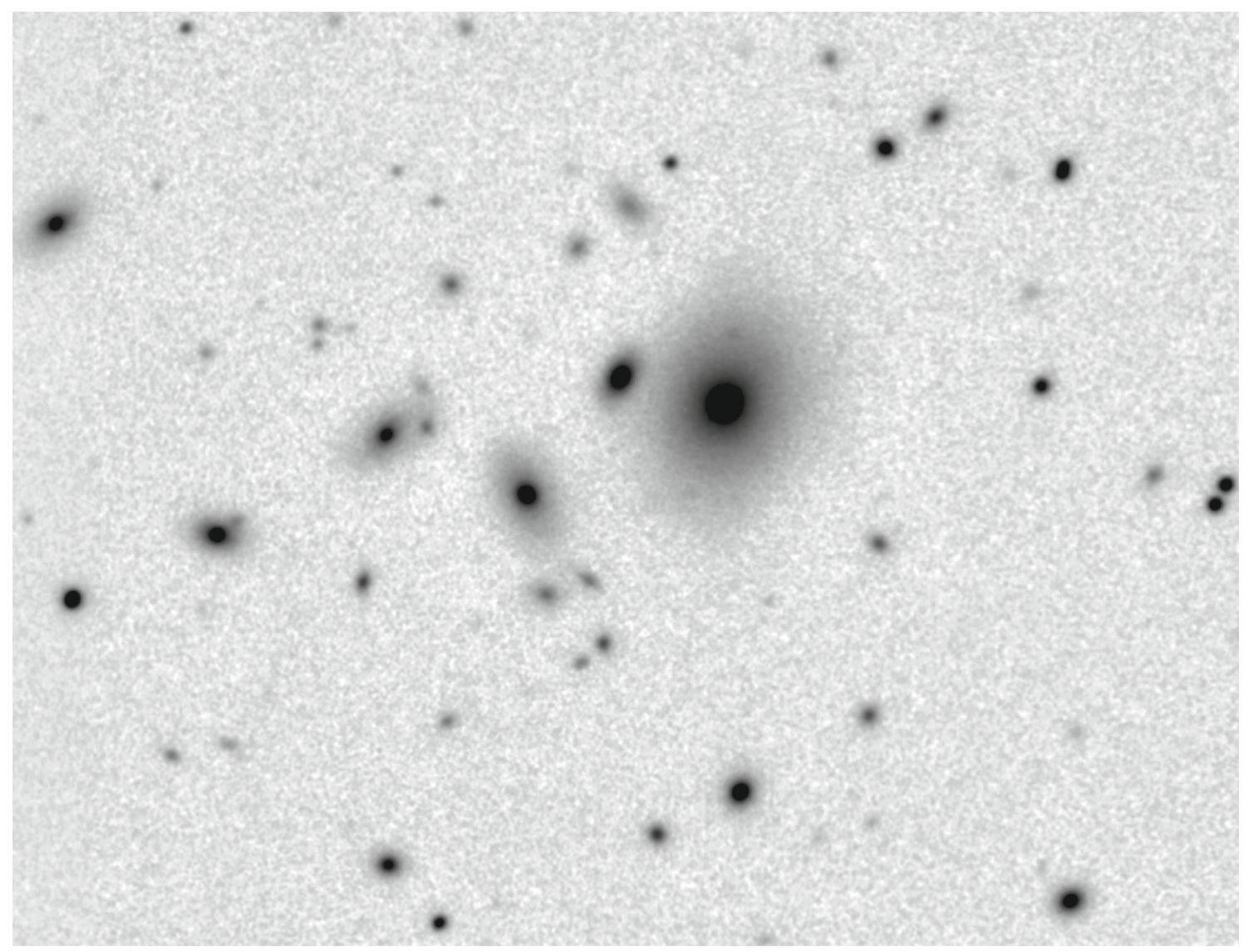

Fig. 2. The central region $\left(2.5^{\prime} \times 2.2^{\prime}\right.$, north is up, east is left $)$ of the cluster A2124 $K$-band mosaic.

$94 \%$ of their width, at the corners of a field of about $0.4 \mathrm{deg}^{2}$. More details about the camera, imaging properties, defects and drawbacks can be found in Dye et al. (2006). Figure 3 shows the angular vs. physical median FWHMs for $J$ - (blue open circles; dashed line) and $K$-band (red full circles; full line). It is seen that more than $50 \%$ of our images have seeing below 1'. 0 , and only three out of 47 co-added mosaics do not meet the quality requirements of the WINGS survey regarding physical size resolution (dotted vertical line; see Paper I). This requirement is needed for reliable morphological classification and surface photometry. A complete and useful log of the observations concerning sky, zero point fluctuations, etc. can be found in the CASU website ${ }^{3}$.

All WFCAM data, including proprietary PI data, are pipeline-processed by the Cambridge Astronomical Survey Unit (CASU) as part of the VISTA Data Flow System (VDFS) development (see, Emerson et al. 2004; Irwin et al. 2004; Hambly et al. 2004, for an overview). The data products of the CASU pipeline include artifact-corrected images, interleaved and stacked as appropriate, confidence maps and catalogues of detected objects (both stellar and extended). All of them are astrometrically and photometrically calibrated with respect to 2MASS. We refer the reader to Irwin et al. (2008) for a detailed description of the pipeline and Hodgkin et al. (2009) for a detailed description and discussion of the photometric calibration. An overview of the pipeline and photometric calibration can also be found in the WFCAM section of the CASU web site ${ }^{4}$, which

\footnotetext{
${ }^{3}$ http://casu.ast.cam.ac.uk/surveys-projects/wfcam/ dataprocessing

4 The web page http://www . ast. cam.ac.uk/ wfcam provides access to the WFCAM raw data and includes detailed description and useful summaries of the observing and reduction processes.
}

contains other quality control plots too, showing, for each night, the variations of the photometric zero-points, the image seeing (FWHM) and sky background level (see Riello \& Irwin 2008, for an overview) that proved very useful for assessing the quality of the data presented in this paper.

\subsection{Pipeline data products}

WFCAM observations are organised in groups, where a group is composed of all the images taken with a given filter, dither and, optionally, micro-step sequence. For all the clusters presented in this paper, observations were taken using a 9-point dithering pattern with a $2 \times 2$ micro-step sequence to partially recover the spatial resolution $^{5}$ (Casali et al. 2007). Micro-stepping is done by shifting the telescope by $n+\frac{1}{2}$ pixels (for the $2 \times 2$ pattern) and interleaving consists of an algorithm that creates an output image that is a regular interwoven pattern of all the input pixels. Some relevant caveats about interleaving for this paper are: i) interleaving does not eliminate bad pixels; ii) the PSF often varies on short enough timescales to lead to unusually "spiky" interleaved PSFs that required additional treatment to deal with. The first issue was addressed by adopting a dithering strategy that allows an efficient rejection of cosmic rays and bad pixels at the stacking phase. We will discuss the second issue in the following section.

Each group is therefore composed of 36 independent MultiExtension FITS files (MEF) with four extensions (one per

\footnotetext{
5 Micro-stepping and interlacing allow scales of $1 / 2$ and $1 / 3$ of the original pixel size, avoiding the PSF under-sampling when good seeing conditions occur. In all our observations the effective pixel size is 0.2 .
} 


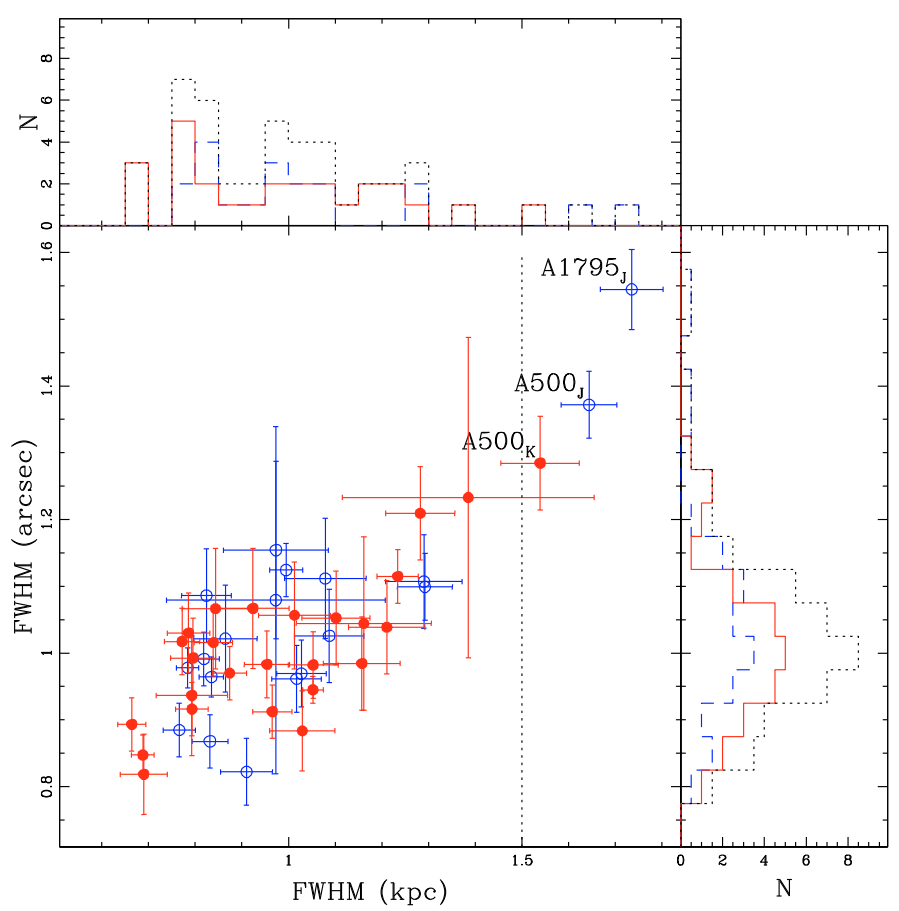

Fig. 3. The distribution of the median FWHMs (angular vs. physical) of all the $J$ - (open blue circle; blue dashed line) and $K$-band (filled red circles; red full line) mosaics: black dotted lines are the total $J$ and $K$ distributions. The error bars show the rms of the median seeing variation of each section of the mosaic, as shown in Fig. 4. Note that a large error bar means that, due to non-photometric nights and/or technical problems with the camera, the FWHM was unstable among the different exposures making up the final mosaic. The dotted vertical line is the limit for quality requirements of WINGS survey spatial physical resolution, needed for reliable morphological classification and surface photometry.

detector). Given that the WFCAM four detectors are separated, in both directions, by $\approx 90 \%$ of their size, it is necessary to take four images (i.e., four MEFs) with appropriate offset in order to survey a contiguous area of about $0.79 \mathrm{deg}^{2}$ (a tile, see Fig. 4). Depending on the cluster and the filter, one or more tiles were observed to achieve the desired magnitude limit.

For each image group the pipeline produces a stacked image from the interleaved $\left(0{ }^{\prime} 2\right.$ sampled) frames coming from each dithering point. Each stacked frame is astrometrically and photometrically calibrated and has an accompanying object catalogue and confidence map generated for it. These data products represent the input for the final stage of the processing which involves:

1. stacking, for each of the four tile positions, all the individual pipeline stacks (typically 2 for $J$-band observations and 4 for $K$-band ones). To avoid confusion we will refer to these images as the (four) final stacks;

2. mosaicking the four final stacks into a single image covering $\approx 0.79 \mathrm{deg}^{2}$.

\subsection{Final stacks and mosaics}

The final stacks are produced using a stand-alone version of the image stacking engine used by the CASU pipeline. The main difference of the stand-alone version is that it uses the object catalogues for further refinement of the WCS (World Coordinate System) offsets that are stored in the FITS headers.
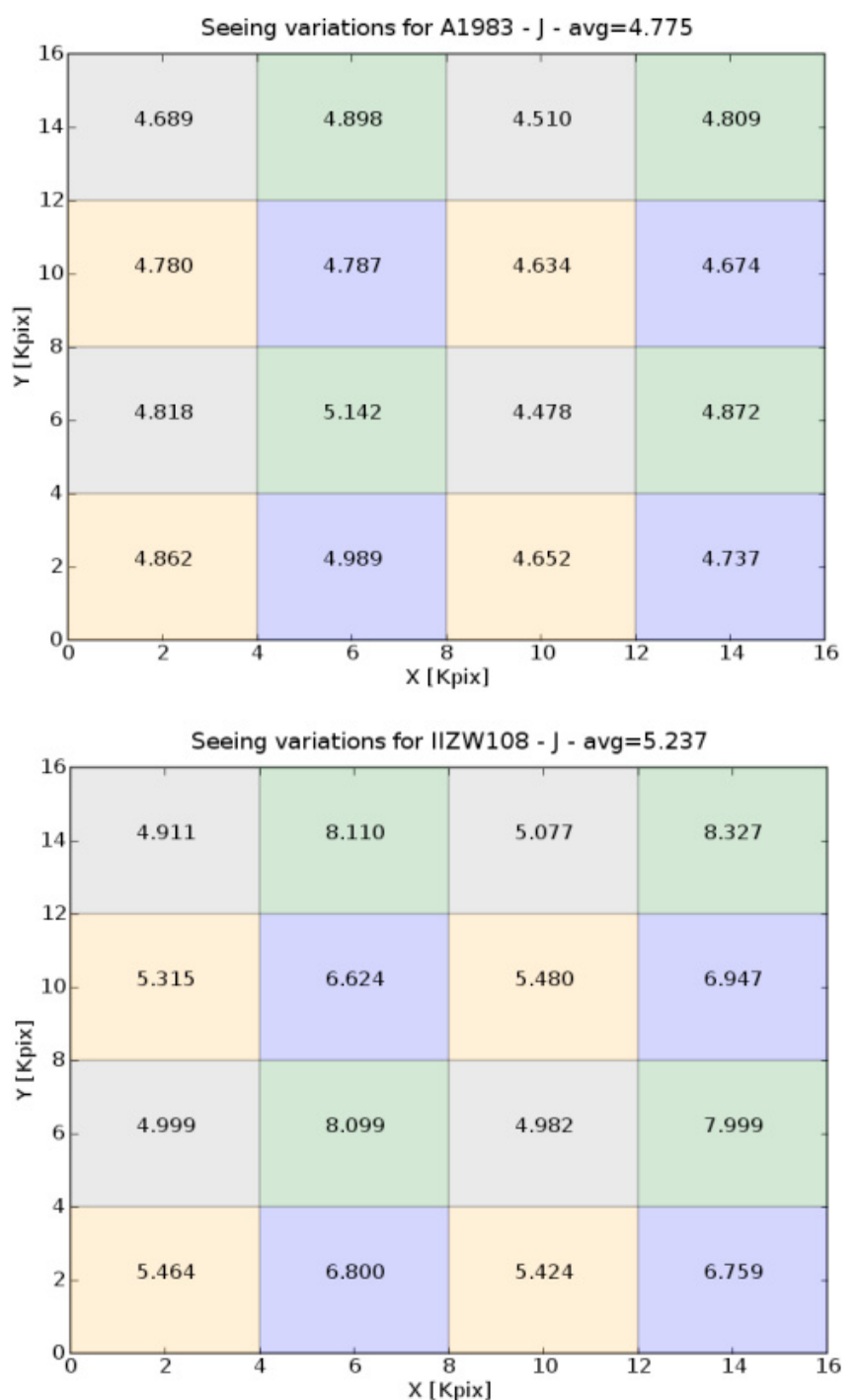

Fig. 4. Due to the WFCAM configuration and the way the observations are carried out, the final mosaics have global PSF variations in a chessboard pattern fashion. The "tile" described in the text, is composed of 4 exposures (i.e., MEFs), of 4 detectors each, which gives the 16 sections of the mosaic shown here (pixel size is 0 '. $^{\prime}$ ). The best and the worst global PSF of our mosaics is presented in the top and the bottom panel, respectively. For each of the 16 sections, the median FWHM (in pixels) of well classified and non saturated stars is reported.

In particular:

1. the alignment of the input images is driven by the WCS of the input frames and then is further refined using the associated object catalogues. The first FITS image in the stacking list is used as a reference and the other images are resampled (using nearest-neighbour interpolation) onto the WCS of the first image;

2. all the images are then scaled at the detector level, using additive corrections, to match the background of the common overlap area;

3. the next step involves rejecting bad pixels using the input confidence maps and an iterative $k$-sigma clipping that uses a sky background-calibrated Poisson noise model for the frame (which uses a robust MAD ${ }^{6}$-based sky noise estimator to define the equivalent RMS noise level);

4. finally, pixels are combined using inverse variance weighting derived from the combination of the input confidence

${ }^{6}$ MAD: median absolute deviation from the median. 
maps and the average noise properties of the image with an additional weighting according to the seeing (i.e. weight $\propto$ seeing $^{-2}$ ).

A two-dimensional non-linear iterative filter was then applied to remove the background from the final stacks. This additional step is required because the stacked frames produced by the pipeline, although corrected for residual reset-anomaly, gain and low-level sky variations, still show background variations $\left(\sigma_{\mathrm{BG}}=0.7 \div 1.2 \mathrm{ADUs}\right)$. Finally we used a variant of the drizzle algorithm (see e.g., Fruchter \& Hook 2002) to remap the original 0. ' 4 pixels that were interleaved into a finer $0 . ' 2$ grid to obtain smoother PSFs (see Sect. 2.1).

The final stacks are eventually combined together to form a mosaic, again using a stand-alone version of the pipeline software. The software uses the photometric zero points associated with each of the four input images to match them photometrically and then project them on the same grid using a drizzle-like algorithm. To reduce the artifacts caused by the poorer image edges, all the input pixels with an associated confidence lower than $50 \%$ were rejected from the final mosaic. Finally the mosaic zero-points are re-measured with respect to 2MASS using the same software used by the CASU pipeline (see Hodgkin et al. 2009), and a final confidence map for the mosaic is produced from the confidence maps of the single stacks.

\section{The photometric catalogs}

After preparing the final photometrically and astrometrically calibrated co-added mosaics (hereafter simply called mosaics) we used a custom pipeline of miscellaneous scripts to generate the source lists. We will use SExtractor's MAG_AUTO (with the default input parameters, Bertin \& Arnouts 1996, see manual for details) as the default magnitude throughout this paper, unless explicitly otherwise mentioned.

Hereafter we present a step-by-step schematic description of the catalogs production:

- selection of a polygon enclosing the reliable region of the mosaic, whose total area is reported in Table 3. In practice, the edges of the mosaic are excluded. We also exclude objects that are dissected by, or are not far enough away from the edges to ensure their photometry is unaffected;

- running the Terapix software WeightWatchers to generate a flag mask, to locate the detections inside the good region and those with confidence $<70 \%$ as deduced from the confidence maps of the final mosaics: we remind the reader that pixels from the single stacks with confidence levels below $50 \%$ were not included in the final mosaic (see previous section);

- preliminary running of SExtractor, to evaluate the FWHM of non saturated stars (CLASS_STAR $>0.95$ ), to choose the detection thresholds, and to quickly check the quality of the mosaics (astrometry, photometry, number counts ...), as explained in Sect. 3.1;

- extraction of several background stamps from the mosaic, useful to prepare a background image for photometric errors estimates with simulations. A collection of synthetic stars and galaxies (30\% of exponential disks) is separately added to the background image, in an attempt to best reproduce the FWHM distribution of the real image. Detection and classification rates of stars and galaxies are computed separately (see Table 3) and are also used to fine tune the SExtractor input parameters (see Sect. 3.1);

- final running of SExtractor with the adjusted input parameters and partitioning of the main output catalog into the catalogs of stars, galaxies and unknown objects. The latter step is achieved here just relying upon SExtractor's CLASS_STAR, which ranges from 0 (galaxies) to 1 (stars), in the following way ${ }^{7}$ :
Stars
CLASS_STAR $\geq 0.8$
Galaxies CLASS_STAR $\leq 0.35$
Unknown $0.35<$ CLASS_STAR $<0.8$;

- since the CLASS_STAR parameter is not reliable enough in all circumstances, in particular when the mosaic is affected by strong seeing variations, a further step is necessary in order to improve the assignment of each object to the right cata$\log$ and to remove from all of them the spurious detections. For this task we used a custom interactive tool which produces various diagnostic plots, and allows us to distinguish between stars and galaxies;

- final "visual" (interactive) cleaning of the mosaic to correct any remaining blatant error both in detection and classification. In this phase the mosaic is displayed with the corresponding markers of stars and galaxies and, by visual inspection, the corrections are made directly on the image, saved on disk and then applied to the catalogs.

In the following sections we will describe in more detail some of the aforementioned steps taken as part of the catalogs production.

\subsection{Source detection and star/Galaxy classification}

The source detection was performed by running SExtractor on the final mosaics, convolved by 2D Gaussian filters of size chosen to be equal to the median FWHM. In most cases, the low level of the background rms obtained with the drizzling techniques of the CASU pipeline, allowed us to use a $1.5 \sigma$ clipping and a minimum area of 20 adjacent pixels as threshold parameters. These parameter values allowed us to simultaneously obtain a small number of spurious detections of stars and galaxies, and deep enough magnitude limits. In general, for each image, the right combination of threshold, minimum area and filter size was chosen relying upon the expected number counts of galaxies/stars and on serendipitous visual inspection of marked detections with the SAO-DS9 display tool. Spurious detections are typically misclassified as galaxies by SExtractor, and usually result from local background fluctuations and spikes or crosstalk of saturated stars.

The preliminary star/galaxy classification was done relying upon SExtractor's stellarity index (CLASS_STAR). The median FWHM of the image to be processed (SEEING_FWHM) is the chief parameter affecting the SExtractor's star/galaxy separation algorithm. The sigma-clipping and filtering detection parameters used in SExtractor are also quite important, since background fluctuations and PSF distortions near the detection threshold can introduce uncertainties correlated with such quantities.

Aiming to test on our mosaics both the detection capability of SExtractor and the reliability of its star/galaxy classifier, we have produced for each cluster a mock image of simulated stars and galaxies (a mix of $70 \%$ spheroidal $r^{1 / 4}$ and of $30 \%$ exponential disks) with a sample background coming from the real image, and trying to match as far as possible the distribution of the stellar FWHM. With ARTDATA package, synthetic stars were modeled using Moffat profiles with $\beta=2.5$. For spheroidal

\footnotetext{
7 In Paper II the galaxies value was 0.2: the difference is due to the intrinsic differences between near-infrared and optical images. These values are estimated using simulations which reproduce the peculiar characteristics of the mosaics.
} 


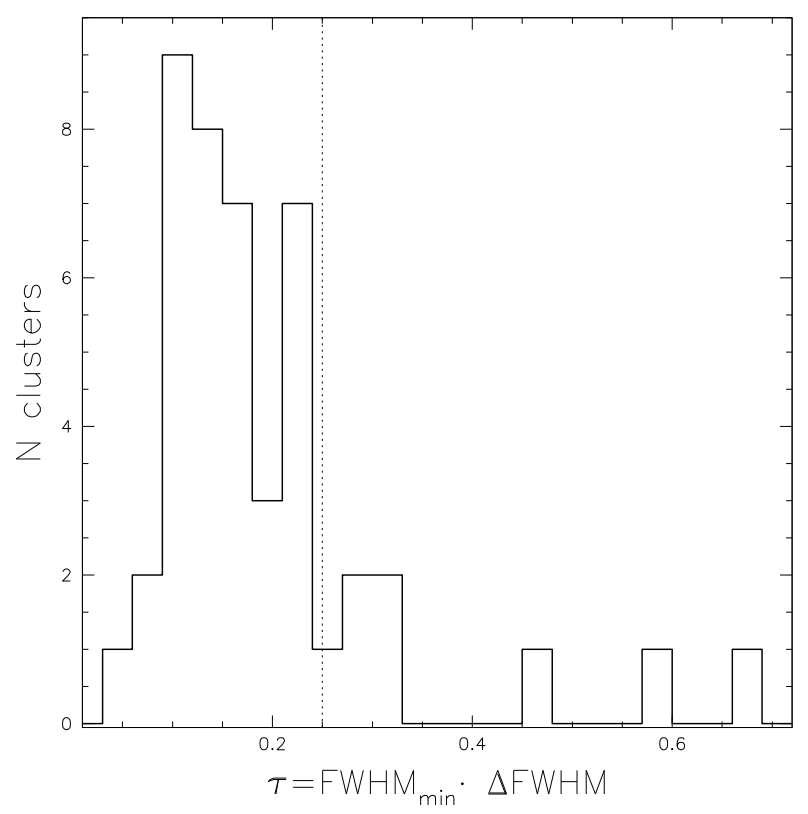

Fig. 5. Distribution of the quantity $\tau=\mathrm{FWHM}_{\mathrm{MIN}} \cdot \Delta \mathrm{FWHM}$ of Eq. (1) for all the near-infrared mosaics. FWHM $_{\max / \min }$ are the maximum and the minimum values of the median FWHM, calculated on the single detectors of the final stacked tiles, as shown in Fig. 4. For all clusters with $\tau<0.25$ (the region delimited by the vertical dotted line) both the star/galaxy classification and the cleaning of the catalogs are quite straightforward.

De Vaucouleurs and disk profiles, intrinsic half-light radii in the range $1-5 \mathrm{kpc}$ and an Euclidean power law $(n=0.3)$ luminosity function were adopted.

Foucaud et al. (2007) claim that a $20 \%$ increase of the value of SEEING_FWHM parameter had to be used to correctly match the synthetic stars added to the images, and this indicates the difficulty SExtractor has to correctly classify stars, at fainter magnitudes, even in good seeing conditions. We further investigated this effect and found that, while the FWHMs of stars produced by the ARTDATA-IRAF package are consistent with the input values, their SExtractor estimates are $\approx 15 \%$ higher. This drawback can be dealt with following the Foucaud et al. (2007) prescriptions of generating mock images with correspondingly diminished input values of the FWHM. The results of these simulations are presented in Sect. 4.1, where we discuss the completeness of the catalogs.

The PSF spatial variation exemplified in Fig. 4 is by far the most important effect which might complicate the correct choice of CLASS_STAR. In fact, while the resulting CLASS_STAR is highly sensitive to the SEEING_FWHM keyword, up to the last release of SExtractor has this keyword fixed for the whole frame. This means that, in case of strong space variations of the FWHM, SExtractor will tend to overestimate the number of stars (galaxies) in the mosaic regions where the local FWHM is greater (lower) than the median value used for source extraction.

In Fig. 5 we present, for all our clusters in both bands, the distribution of the quantity:

$\tau=\mathrm{FWHM}_{\mathrm{MIN}} \cdot\left(\mathrm{FWHM}_{\mathrm{MAX}}-\mathrm{FWHM}_{\mathrm{MIN}}\right)$

where FWHM_max/min are the maximum and the minimum median FWHM of the single stacked detectors, respectively, as determined from grids like those in Fig. 3. The value of $\tau$ is a quality and stability indicator of the PSF for complete mosaics and all the observations collected during a given night. Figure 5 shows that the majority of our clusters have relatively low values (high

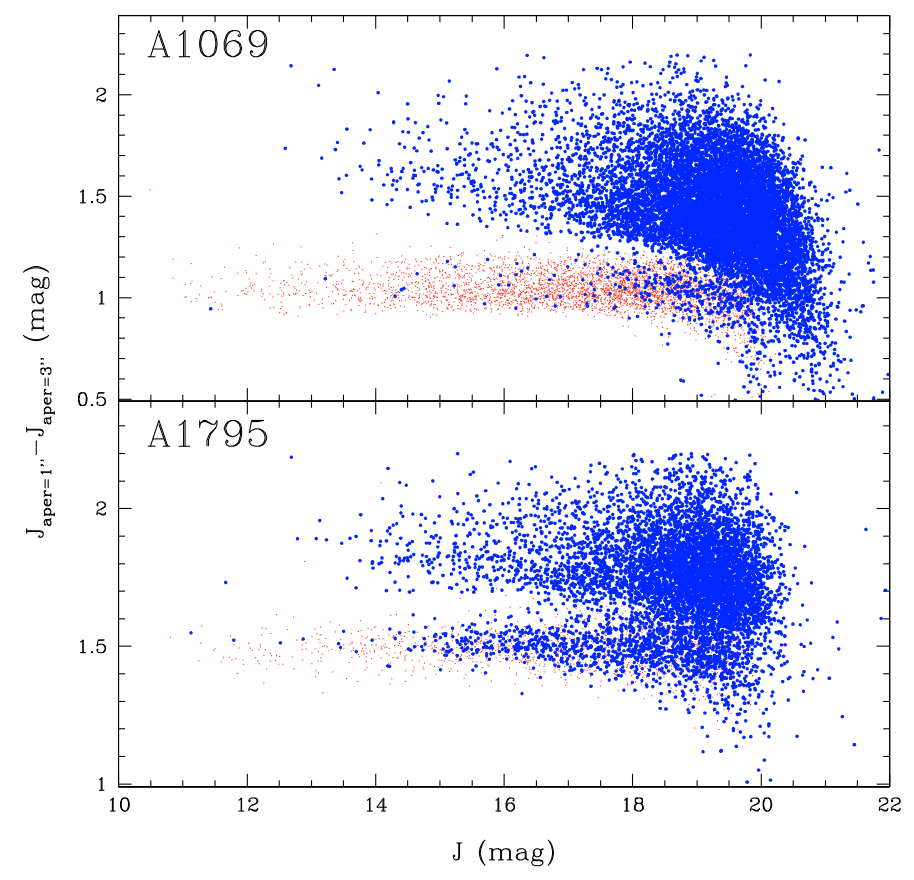

Fig. 6. Plot of the difference between two aperture magnitudes (1 arcsec-3 arcsec diameter) vs. total magnitude for galaxies (blue bold dots) and stars (red light dots) as classified by SExtractor before any interactive cleaning. In this diagram the star and galaxy loci turn out to be well apart down to $J \approx 19.0$. In the good cases (top panel: A1069, with quite stable seeing during observations, $\tau=0.21$ ), only a few galaxies are misclassified, and even at bright magnitudes. Alternatively, in cases of strong seeing variations (bottom panel: A1795, $\tau=0.32$ ), many more misclassifications of galaxies may be found (bold dots in the locus of stars).

quality) of this quantity ( $\tau<0.25$; marked by the vertical dotted line). In clusters with $\tau>0.25$ the choice of the SEEING_FWHM keyword and other parameters is very difficult, due to many misclassifications being found, and the use of the interactive cleaning procedure becomes fundamental to generate reliable stars and galaxies catalogs (see Fig. 6).

\subsection{Interactive cleaning}

Spurious detections are most frequently found along the overlapping regions of the detectors, along the mosaic edges (which are excluded by the initial polygon mask) and in (usually limited) regions of high background fluctuations. Cross-talk due to saturated stars is another cause of spurious objects. These false detections can be found at fixed distances (in symmetric positions) from the saturated objects along the read-out direction of the detector, and have a characteristic "doughnut" shape (see, i.e., Dye et al. 2006). It was not possible to safely detect and delete them automatically, as their number and occurrence is not simply correlated with the peak intensity and the area of the saturated object, and in the final mosaic they can be found either in the $X$ or in the $Y$ direction. On the other hand, by exploiting the SExtractor classification capabilities, it was found that only the brightest ones are misclassified as galaxies, and most of them are easily deleted by the interactive cleaning procedure described below. Since the automated pipeline produces a separate catalog of the saturated stars, during the final visual check, we highlight them, allowing easy identification and deletion from the final catalogs of their associated cross-talk false detections. A negligible fraction of these objects, depending on the number 
Table 2. Example of entries of $J$ and $K$ photometric catalogs.

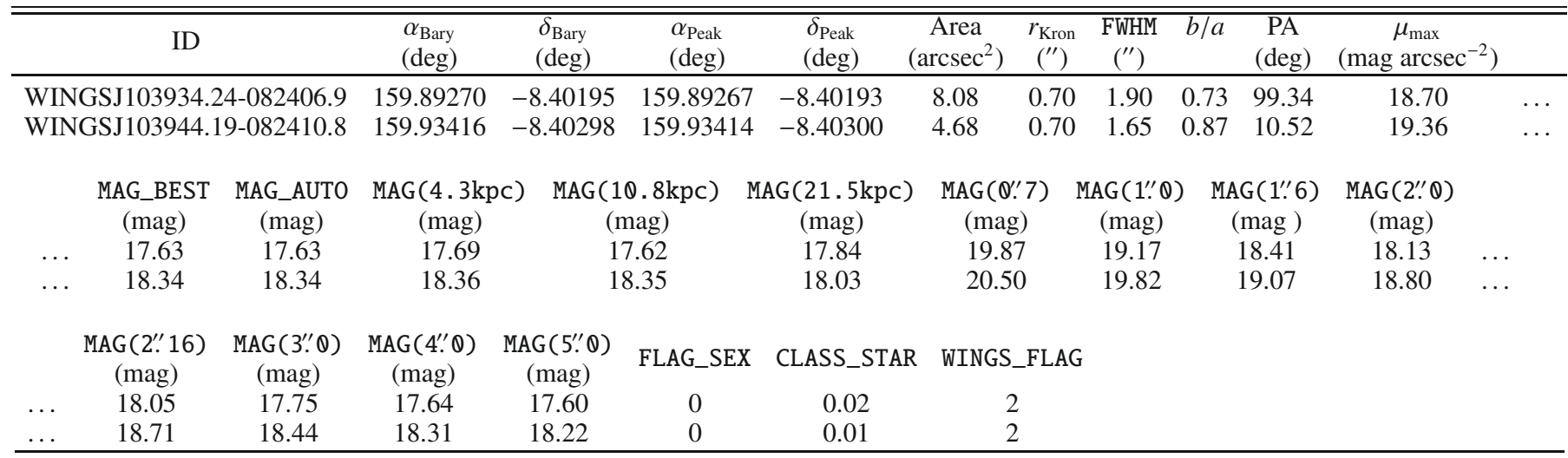

The full table, for each cluster and filter, is available in electronic form at the CDS.

of saturated stars in the mosaic, may remain after this last check, but are mainly classified as "unknown" sources.

Since the number of objects is too large for any individual analysis, we purged spurious detections and star/galaxy misclassifications with our interactive tool, which generates several plots of different combinations of parameters, like:

- $\mu_{\max }$ vs. $J, K$, where $\mu_{\max }$ is the surface brightness of the brightest pixel of an object and $J, K$ is the total apparent magnitude MAG_AUTO;

- J, $K$ vs. $\log _{10}($ Isophotal Area), where Isophotal Area is the area in pixels of each object at the threshold level;

- $\log$ (FWHM) vs. $\log _{10}$ (Isophotal Area);

- $J, K\left(d \leq 1^{\prime \prime}\right)-J, K\left(d \leq 3^{\prime \prime}\right)$ vs. $J, K$, where $d$ is the aperture diameter.

In all these diagrams, the stars populate a rather narrow and well defined region, while galaxies are more spread throughout the plane (see also, Paper II). As an example, in Fig. 6 a plot of the difference between two aperture magnitudes ( 1 arcsec- 3 arcsec diameter) vs. total $J$ magnitude for galaxies (blue bold dots) and stars (red light dots) before any interactive cleaning, is presented. It is apparent that for the cluster A1795, due to seeing variations and background conditions, a considerable amount of objects with CLASS_STAR typical of galaxies actually populate the region of stars. In the case of A1069, the more stable PSF causes a better classification. Still, at faint magnitudes, a non-negligible amount of misclassifications is left. Thus, a further step turns out to be necessary in any case to clean the catalogs. To this aim, the outliers and/or misclassifications highlighted by these diagrams are interactively selected, and, if wanted, the cleaning pipeline shows a tile-mosaic of these objects for visual inspection to easily select spurious detections. Then, simple commands allow us to look at their location in the original mosaics, list their parameters from the catalog, delete them or change their classification.

At the end of this process the degree of misclassification of relatively bright sources $(J<18.5$ and $K<18.0)$ is less than $1 \%$, that is practically negligible (see Sect. 4.1). Of course, going to fainter and smaller objects the regions occupied by stars and galaxies start to mix up, making the classification more and more unreliable.

The published catalogs will be regularly updated to correct for any newly found spurious objects and/or misclassifications, therefore users are encouraged to rely upon the latest available version of the catalogs.

\subsection{Catalogs description}

In Table 2 we present an example of the entries in the nearinfrared photometric catalogs. The parameters stored for each object are the following (in parenthesis we give the name of the SExtractor's output parameter):

- ID: objects internal identification, it is unique for all catalogs of the WINGS survey.

- $\left(\alpha_{\text {Bary }}, \delta_{\text {Bary }}\right)$ : equatorial coordinates (J2000.0) of the barycenter.

- $\left(\alpha_{\text {Peak }}, \delta_{\text {Peak }}\right)$ : equatorial coordinates (J2000.0) of the brightest pixel.

- Area: area at the detection threshold.

- $r_{\text {Kron }}$ : Kron radius used to compute the MAG_AUTO magnitude.

- FWHM: full width at half maximum assuming a Gaussian core as calculated by SExtractor.

- $b / a$ : axis ratio of the source.

- PA: position angle of the major axis (North $=0^{\circ}$, measured counter-clockwise).

- $\mu_{\max }$ : surface brightness of the brightest pixel.

- MAG_BEST: SExtractor's best total magnitude estimate.

- MAG_AUTO: SExtractor's Kron (total) aperture magnitude.

- MAG(4.3 kpc), MAG(10.8 kpc), MAG(21.5 kpc): magnitudes within apertures of diameter $4.3 \mathrm{kpc}, 10.8 \mathrm{kpc}$ and $21.5 \mathrm{kpc}$, respectively, measured at the clusters' redshift. Since we will adopt in future papers of the survey $H_{0}=70$ (at variance with Paper II, where $H_{0}=75$ has been used), the apertures in kpc are slightly different from those given in Paper II (4/10/20 kpc): however, the value in arcseconds remains the same.

- MAG( $\left.0^{\prime \prime} 7\right), \operatorname{MAG}\left(1{ }^{\prime \prime} \otimes\right), \operatorname{MAG}\left(1^{\prime \prime} \cdot 6\right)$. . . : magnitudes measured within 8 different fixed apertures ${ }^{8}$ in arcsec.

- FLAG_SEX: SExtractor's FLAG keyword.

8 Three of these fixed apertures have been chosen to match the multifiber spectroscopy of the WINGS fields. 1'. 6 is the projected diameter of the fibers using the Autofib2@WHT, while using the 2dF@AAT results in a diameter that varies radially in the field from 2 '. 16 in the center to $2{ }^{\prime \prime} 0$ at the edges. 
Table 3. WINGS-NIR clusters sample with relevant useful quantities.

\begin{tabular}{|c|c|c|c|c|c|c|c|c|c|c|c|}
\hline \multirow[t]{2}{*}{ Cluster } & \multirow{2}{*}{$\begin{array}{c}\text { RA } \\
\text { hh:mm:ss }\end{array}$} & \multirow{2}{*}{$\begin{array}{c}\text { Dec } \\
\text { dd:mm:ss }\end{array}$} & \multirow[t]{2}{*}{ Redshift } & \multirow{2}{*}{$\begin{array}{l}L_{\mathrm{x}} / 10^{44} \\
\mathrm{erg} \mathrm{s}^{-1}\end{array}$} & \multirow{2}{*}{$\begin{array}{l}\text { Pixel } \\
\text { kpc }\end{array}$} & \multirow[t]{2}{*}{ RUN } & \multicolumn{2}{|c|}{ 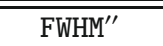 } & \multirow[t]{2}{*}{ Stacks } & \multicolumn{2}{|c|}{ Total area } \\
\hline & & & & & & & $\min$ & $\max$ & & $\mathrm{deg}^{2}$ & $\mathrm{Mpc}^{2}$ \\
\hline A119 & $10: 39: 43.4$ & $-08: 41: 12.4$ & 0.0442 & 1.65 & 0.174 & $05 \mathrm{~B}-K$ & 0.79 & 0.88 & 16 & 0.781 & 7.66 \\
\hline A376 & $2: 46: 03.9$ & $+36: 54: 19.2$ & 0.0484 & 0.71 & 0.190 & $05 \mathrm{~B}-J$ & 1.05 & 1.17 & 8 & .777 & 9.09 \\
\hline A500 & $04: 38: 52.5$ & $-22: 06: 39.0$ & 0.0670 & 0.72 & 0.256 & $\begin{array}{l}05 \mathrm{~B}-J \\
05 \mathrm{~B}-K\end{array}$ & $\begin{array}{l}1.28 \\
1.21\end{array}$ & $\begin{array}{l}1.44 \\
1.42\end{array}$ & $\begin{array}{c}8 \\
16\end{array}$ & 0.776 & 16.48 \\
\hline A602 & 075326.6 & +292134.5 & 0.0619 & 0.57 & 0.238 & $06 \mathrm{~B}-K$ & 0.88 & 0.94 & 12 & 0.778 & 14.29 \\
\hline \multirow{2}{*}{ A957x } & \multirow{2}{*}{$10: 13: 38.3$} & & $\theta$ & 0 & 0172 & $05 \mathrm{~A}-J$ & 1.02 & 1.47 & 8 & 0.779 & 7.47 \\
\hline & & $-00: 55: 31.3$ & 0 & 0.40 & $0.1 / 2$ & $06 \mathrm{~B}-K$ & 0.75 & 0.92 & 8 & 0.782 & 7.50 \\
\hline 170 & 7 & $10 \cdot 4$ & & 077 & $0 ?$ & $06 \mathrm{~B}-J$ & 0.91 & 1.11 & 8 & 0.777 & 13.09 \\
\hline A9I0 & ZJ. & .2 & & 0.11 & 0.228 & $05 \mathrm{~A}-K$ & 1.06 & 1.26 & 16 & 0.775 & 13.05 \\
\hline 1060 & 1.30 .434 & 08.4 & 0 & 0,48 & 250 & $05 \mathrm{~A}-J$ & 0.98 & 1.19 & 8 & 0.783 & $586>$ \\
\hline A1009 & 43.4 & .4 & 0 & 0.48 & 0.250 & $05 \mathrm{~A}-K$ & 0.88 & 1.12 & 16 & 0.782 & 50 \\
\hline A1291 & 232 & 0 & & 022 & 0206 & 06B-J & 0.80 & 0.93 & 8 & 78 & 070 \\
\hline A1291 & 25.2 & & 1 & 0.22 & 0.200 & $05 \mathrm{~A}-K$ & 0.99 & 1.15 & 16 & 18 & 0.10 \\
\hline 16210 & $.57 .52,6$ & & & & ? & $05 \mathrm{~A}-J$ & 0.89 & 1.15 & 8 & 0.781 & \\
\hline Al63la & $12: 52: 52.6$ & .8 & 2 & 0.31 & 0.182 & $05 \mathrm{~A}-K$ & 0.82 & 1.10 & 16 & 0.780 & 8.38 \\
\hline 1644 & 12.52 .526 & 15 & & 180 & 0186 & $06 \mathrm{~B}-J$ & 0.88 & 0.98 & 8 & 0.778 & 8.72 \\
\hline A1044 & 12.52 .52 .0 & -15 & $0.0^{2}+x^{2}$ & 1.80 & 0.180 & $05 \mathrm{~A}-K$ & 0.89 & 1.15 & 16 & 0.772 & 8.65 \\
\hline 1705 & 2.48 .525 & $26 \cdot 3$ & 15 & 567 & $0 \tau-2+$ & $05 \mathrm{~A}-J$ & 1.42 & 1.65 & 8 & 0.780 & 14.56 \\
\hline A1795 & $13: 48.52 .5$ & $+20.35: 34.0$ & 0.0025 & $5.0 \%$ & 0.240 & $05 \mathrm{~A}-K$ & 0.95 & 1.57 & 8 & 0.387 & 7.22 \\
\hline A 1831 & $13 \cdot 50 \cdot 151$ & +27.58 .345 & 00615 & 0.97 & 0238 & $06 \mathrm{~B}-J$ & 0.72 & 0.91 & 12 & 0.778 & \\
\hline A1831 & :59:15.1 & $+21: 58: 34.3$ & 0.0615 & 0.91 & 0.238 & $05 \mathrm{~A}-K$ & 0.93 & 1.07 & 16 & 0.779 & 23 \\
\hline 1002 & 14.57 & 16 & 26 & 024 & 17 & $06 \mathrm{~B}-J$ & 0.90 & 1.03 & 8 & 0.779 & 7.47 \\
\hline A1983 & $14: 52: 55.3$ & $+10: 4$ & 0.0436 & 0.24 & $0.1 / 2$ & $05 \mathrm{~A}-K$ & 0.79 & 0.98 & 16 & 0.772 & 7.40 \\
\hline A1991 & $14: 52: 55.3$ & $+16: 42: 10.6$ & 0.0587 & 0.69 & 0.228 & $05 \mathrm{~A}-J$ & 0.88 & 1.00 & 8 & 0.779 & 13.12 \\
\hline A 2107 & 390 & 0 & 00 & 056 & 0162 & $06 \mathrm{~A}-J$ & 0.95 & 1.19 & 8 & 0.778 & 6.62 \\
\hline 月2101 & 39.0 & .0 & $0.0^{2}+2$ & 0.56 & & $05 \mathrm{~A}-K$ & 0.85 & 1.03 & 16 & 0.748 & 6.36 \\
\hline A2 2124 & 0 & 9 & 6 & 069 & 252 & $05 \mathrm{~A}-J$ & 1.38 & 1.18 & 8 & 0.780 & 16.05 \\
\hline 112124 & .0 & .9 & 0 & & .252 & $06 \mathrm{~A}-K$ & 0.80 & 1.03 & 16 & 0.782 & 16.09 \\
\hline A2149 & $16: 01.28 .1$ & $+53: 56: 50.4$ & 0.0679 & 0.42 & .260 & $06 \mathrm{~A}-K$ & 0.79 & 0.98 & 20 & 0.781 & 17.11 \\
\hline A2 160 & $16 \cdot 13$. & + & 5586 & 023 & 6 & $06 \mathrm{~A}-J$ & 0.89 & 1.03 & 8 & 0.780 & 12.91 \\
\hline A2169 & $16: 13$ & + & 586 & 23 & 0.226 & $05 \mathrm{~A}-K$ & 0.83 & 0.96 & 16 & 0.771 & 12.76 \\
\hline A2382 & $21: 51: 55.6$ & $-15: 42: 21.3$ & 0.0618 & 0.46 & 0.238 & $05 \mathrm{~A}-K$ & 0.89 & 1.25 & 16 & 0.770 & 14.13 \\
\hline A2399 & $21: 57: 01.7$ & $-07: 50: 22.0$ & 0.0579 & 0.51 & 0.224 & $05 \mathrm{~B}-K$ & 0.91 & 1.14 & 16 & 0.781 & 12.70 \\
\hline A2457 & $22: 35: 40.8$ & $+01: 29: 05.9$ & 0.0594 & 0.73 & 0.230 & $05 \mathrm{~B}-K$ & 0.84 & 1.00 & 16 & 0.780 & 13.37 \\
\hline A2572a & $23: 17: 12.0$ & $+18: 42: 04.7$ & 0.0403 & 0.52 & 0.160 & $06 \mathrm{~A}-K$ & 0.79 & 0.94 & 16 & 0.781 & 6.48 \\
\hline A2589 & $23: 23: 57.5$ & $+16: 46: 38.3$ & 0.0414 & 0.95 & 0.164 & $06 \mathrm{~A}-K$ & 0.87 & 1.07 & 16 & 0.781 & 6.81 \\
\hline U7W108 & 21.13 .550 & $+02 \cdot 3$ & 0403 & 112 & 102 & $05 \mathrm{~B}-J$ & 0.98 & 1.66 & 8 & 0.777 & 9.28 \\
\hline $112 \mathrm{~W} 108$ & .9 & + & & .12 & 192 & $05 \mathrm{~A}-K$ & 0.87 & 1.03 & 16 & 0.780 & 9.32 \\
\hline$M K \mathrm{~V}$ & 1 & & & 37 & & $06 \mathrm{~B}-J$ & 0.92 & 1.03 & 8 & 0.780 & 3 \\
\hline MKW $3 \mathrm{~S}$ & $15: 21: 51.9$ & $+0 /: 42: 32.1$ & & 1.31 & 0 & $06 \mathrm{~A}-K$ & 0.88 & 1.06 & 16 & 0.781 & 1.83 \\
\hline DY $>$ & & & & & & $05 \mathrm{~B}-J$ & 0.91 & 1.22 & 8 & 0.779 & 9.30 \\
\hline КАIUZZ & 1 & +30.31 .25 .9 & & & & $06 \mathrm{~B}-K$ & 0.80 & 0.95 & 8 & 0.782 & 9.34 \\
\hline RX1740 & $17: 40: 32.1$ & $+35: 38: 46.1$ & 0.0430 & 0.26 & 0.170 & $05 \mathrm{~A}-K$ & 0.91 & 1.23 & 12 & 0.778 & 7.28 \\
\hline Z8338 & $18: 11: 05.2$ & $+49: 54: 33.7$ & 0.0473 & 0.40 & 0.186 & 06A- $J$ & $\begin{array}{l}0.80 \\
070\end{array}$ & 0.95 & 8 & 0.778 & 8.72 \\
\hline & & & & & & $5 \mathrm{~A}-K$ & 0.79 & 0.92 & 16 & & \\
\hline
\end{tabular}

RA, Dec: coordinates of the Brightest Cluster Galaxy.

Redshift: from NED.

$L_{\mathrm{x}}$ : X-ray luminosity.

Pixel: pixel size in $\mathrm{kpc}$ at the given redshift.

RUN: semester and passband of observation.

FWHM: minimum and maximum estimation as shown in Fig. 4.

Stacks: Interleave Stacks per mosaic used.

Total area: the effective area of the mosaic. 
- CLASS_STAR: SExtractor's stellarity index.

- WINGS_FLAG: summarizing flag column reporting pipeline classification and photometric quality of the objects, using the following prescription:

WINGS_FLAG $=a_{1}+2 a_{2}+4 a_{3}+8 a_{4}+16 a_{5}+32 a_{6}$

$a_{1}=1$ if classified as star;

$a_{2}=1$ if classified as galaxy;

$a_{3}=1$ if classified as unknown;

$a_{4}=1$ if weakly affected by neighbouring halo;

$a_{5}=1$ if strongly affected by neighbouring halo;

$a_{6}=1$ if in an area of confidence $<70 \%$;

where the classification is done relying upon our custom interactive tools, the neighbouring halo can be due to saturated stars or nearby extended objects, and the confidence level is extracted from the final confidence maps generated by CASU pipeline (see Sect. 2.2).

The complete/updated list of stored parameters, together with SExtractor configuration files, are available on the WINGS website http://web.oapd.inaf.it/wings/, while the exact definition of each parameter can be found in the SExtractor manual (Bertin \& Arnouts 1996). The photometric catalogs, in the form of Table 2 are available in electronic form at the CDS. Note that the same caveats and/or qualifications given in Paper II hold for these photometric and geometric parameters.

The final version of all WINGS survey catalogs will be a comprehensive cross-matched source-list, allowing multiple criteria queries with Web based tools and Euro-VO facilities, and simple identification of objects which have measurements and calculated quantities belonging to different branches of the survey ( $U, B, V, J, K$ photometry, spectroscopy).

\section{Data quality}

As already mentioned in previous sections, the bulk of the reduction has been performed at CASU with the custom designed pipelines for UKIRT-WFCAM. Hereafter we present the results of the quality tests performed by our pipeline on source extraction and classification, both in real and simulated mosaics.

\subsection{Completeness}

Figure 7 illustrates the global detection and successful classification rates (for both stars and galaxies) evaluated running SExtractor on the mock images, as explained in the previous Sect. 3.1 (see also Table 3). Full and dashed lines are the fraction of the total input objects, detected and correctly classified, respectively. The $90 \%$ level of successful classification is reached at $J=19.0$ and $K=18.2$ for stars, while the limits become $J=19.5$ and $K=18.5$ for extended sources. Moreover, the $90 \%$ detection rate limits for galaxies is reached at $J=20.5$ and $K=19.4$. In Table 3 we report the detection and classification $90 \%$ completeness limits for both stars and galaxies for each cluster and band, as extracted from our simulations.

It is clear that SExtractor is able to classify correctly almost all the galaxies detected in simulations (apart from rare and very compact objects), while this is not true for point-like sources. This is likely due to the seeing variations across the mosaics (which in a few cases is relevant, see Figs. 4, 5). In Fig. 7, the three dotted horizontal lines mark the $95 \%, 90 \%$ and $75 \%$ completeness limits of our survey, while the red dasheddotted line in the upper panels represent the fraction of stars misclassified as galaxies when relying only upon SExtractor's CLASS_STAR. It can be seen that for $J, K>18.0$ the frequency

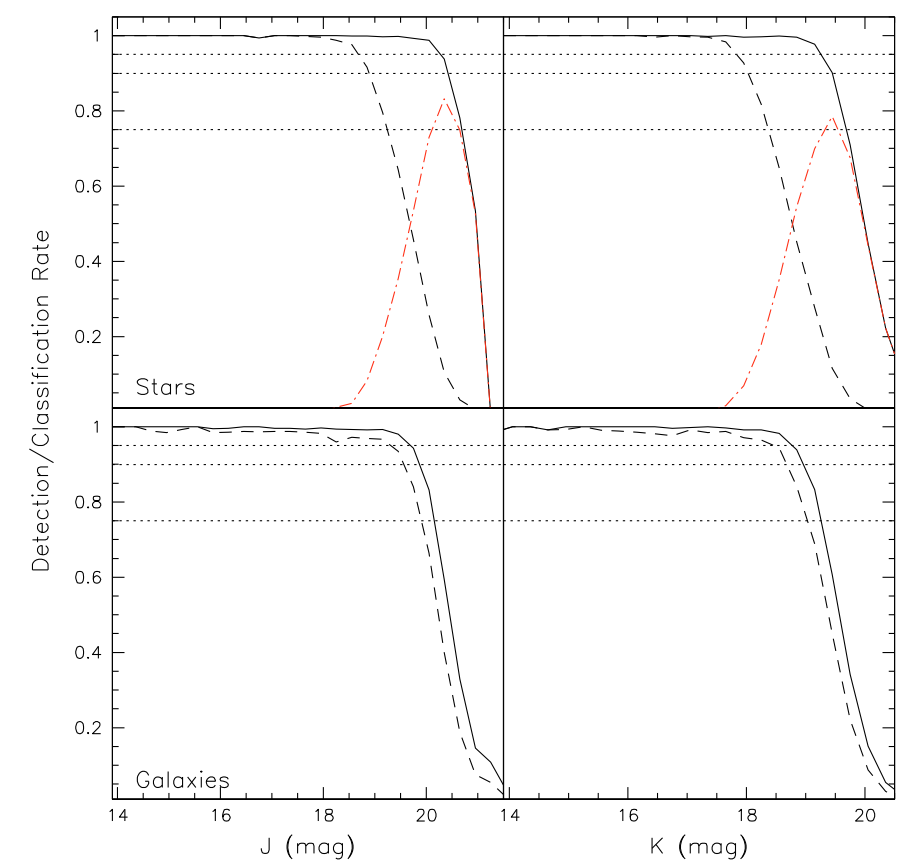

Fig. 7. The global average detection (full lines) and successful classification (dashed lines) rates, obtained from simulations for the WINGSNIR survey in the $J$ - (left panels) and $K$-band (right panels). The dotted lines represent the 95, 90 and 75 percent of completeness. The dotteddashed line in the upper panels represent the fraction of stars misclassified as galaxies by SExtractor. The bottom panels show that, even at bright magnitudes, some galaxies (a small fraction, indeed) are missclassified as stars. This is likely due to the wide range of effective radii adopted in the simulations, which can sometime produce unrealistically compact luminosity profiles. In real mosaics, such misclassifications are easily corrected with our interactive tool.

of such misclassification becomes relevant (see also Fig. 6) and rapidly increases. However, we have to consider that, for faint magnitudes, the galaxy population becomes gradually dominant (field galaxies) and the contribution to the galaxy counts coming from the (usually small) fraction of misclassified stars should be in any case negligible (see, for e.g., Berta et al. 2006).

An empirical (a posteriori) check of completeness can be performed, for each cluster in each band, comparing the number counts of classified objects in our catalogs, with the expected number counts of field galaxies and stars. In Fig. 8 we show representative cases for $J$ - (left panels) and $K$-band (right panels). The red dashed lines are the expected number counts of stars in the area of the cluster, calculated with the TRILEGAL code (Girardi et al. 2005) in the photometric system of WFCAM. The black solid lines are the number counts of field galaxies taken from the UKIDSS Ultra Deep Survey (see, Hartley et al. 2008; Lawrence et al. 2007). The open red and the full blue dots are the WINGS number counts for stars and galaxies, respectively, as calculated from our source lists, with their Poissonian error bars. In general, the agreement between the expected star counts and our catalogs is outstanding, confirming the excellent performance of the TRILEGAL code as a model of the Galaxy. However, for 6 clusters out of 28, we noticed an excess in the real star counts at magnitudes brighter than 18 (see for instance A1644 in Fig. 8). Since all these clusters are found to lay approximately in the direction of the center of the Galaxy (albeit with $|b| \geq 20^{\circ}$ ), where a higher fraction of halo stars is encountered, we assume that the model might slightly break down in this direction. Our galaxy counts agree with the field number counts 


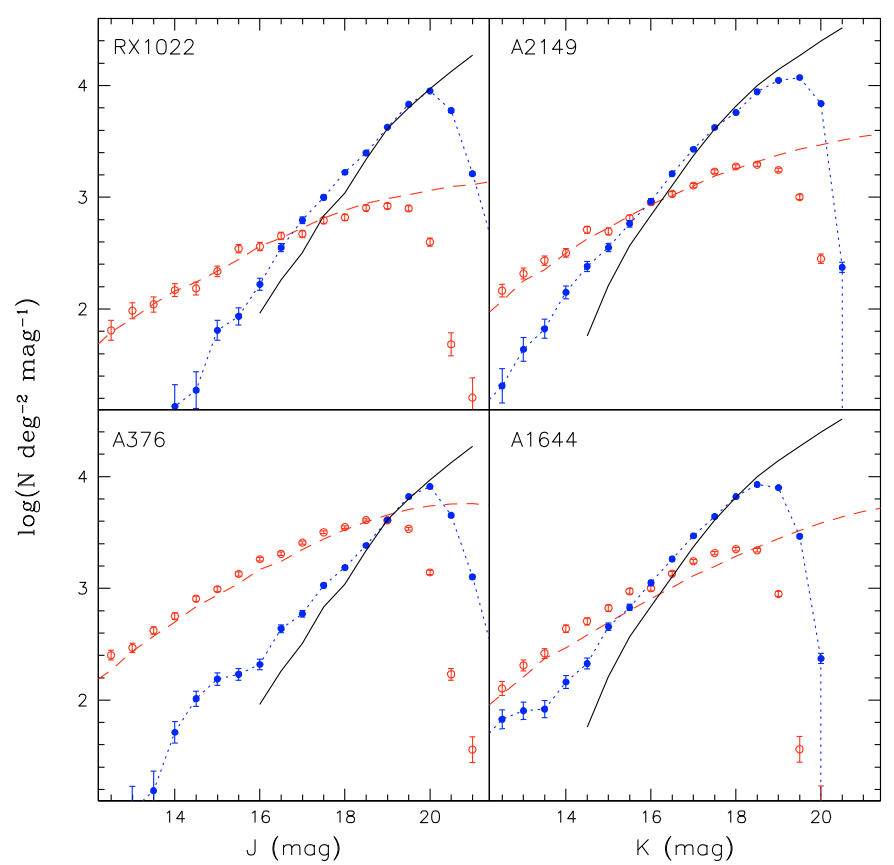

Fig. 8. Number counts in $J$ - (left panels) and $K$-band (right panels) compared with TRILEGAL models of the Galactic stellar distribution from Girardi et al. (2005) (red dashed lines) and field galaxy counts from UKIDSS-UDS Hartley et al. (2008) (solid black lines). The blue full and the red open dots are the galaxy and stars number counts of the corresponding WINGS catalogs. Poissonian error bars are mostly of the dimension of the symbols.

only at faint magnitudes, as expected, showing the presence of cluster galaxies at brighter magnitudes.

The excess of number counts shown in Fig. 8, revealing the cluster members, can also be seen in Fig. 9, where the number counts of all the detected sources (full line), is compared with the cumulative (stars+field galaxies) counts expected for the area of the clusters (dashed line). The approximate magnitude where the turnover in the counts occur is a good estimate of the observed completeness limit, and is reported in Table 3, together with the theoretical magnitude limit (dotted-dashed vertical lines) calculated with the following formula:

$m_{\mathrm{lim}}=\mathrm{ZPT}-2.5 \log _{10}\left[v\left(\sigma_{\mathrm{BG}}+1\right) A_{\mathrm{min}}\right]$

where $v$ is the relative threshold cut in units of background rms $\left(\sigma_{\mathrm{BG}}\right), A_{\min }$ is the minimum number of contiguous pixels required for detection and ZPT is the mosaic zero point magnitude, normalized to one second exposure time and airmass corrected:

$\mathrm{ZPT}=\mathrm{MAGZPT}+2.5 \log _{10}(5)-(\chi-1) \cdot k$

where MAGZPT is the zero-point magnitude keyword found in the FITS header of the mosaic, resulting from the calibration with 2 MASS performed at CASU. The second term refers to the WFCAM 5s exposure time, the $\chi$ and $k$ variables are the average airmass and extinction (keywords AIRMASS and EXTINCT in FITS file), respectively.

This theoretical limit corresponds to the magnitude of an object consisting of $A_{\min }$ contiguous pixels with ADU counts of $v\left(\sigma_{\mathrm{BG}}+1\right)$, and gives an idea of the overall depth of the mosaic imaging, since it links photometric and detection properties together. The dash-dotted vertical lines in Fig. 9 show that these limiting magnitude values are quite consistent with the faintest detected objects.

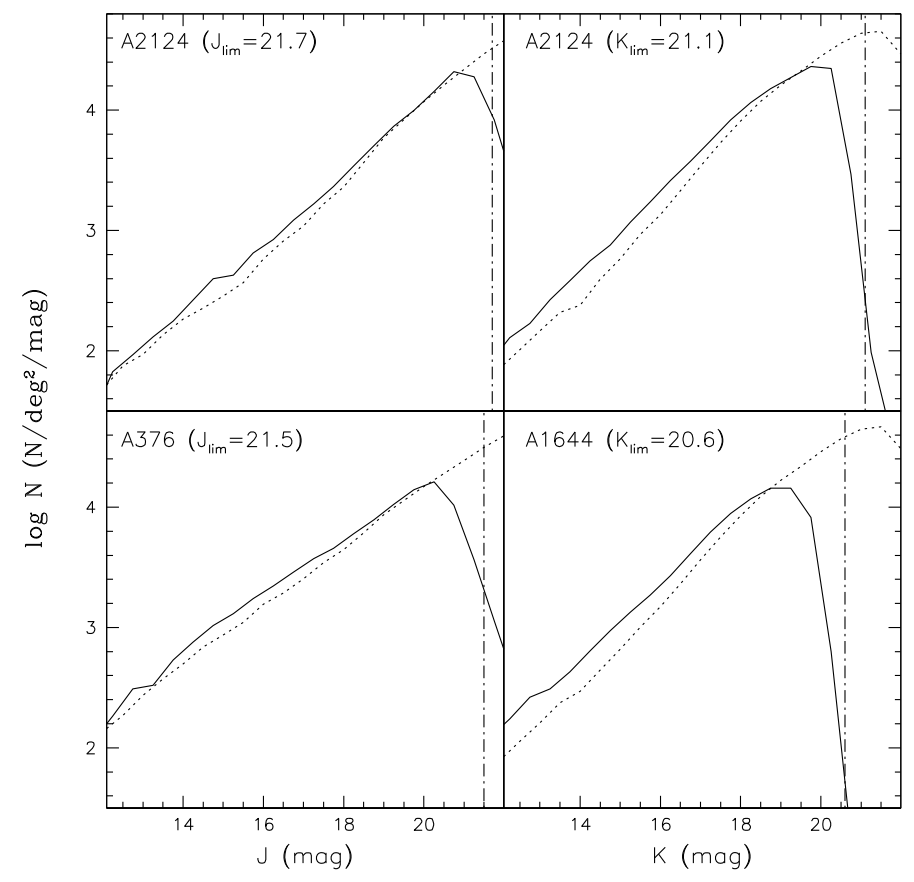

Fig. 9. Magnitude counts of all detections in $J$ - (left panels) and $K$-band (right panels), for the deepest (upper panels) and the shallowest (bottom panels) cluster mosaics. The dash-dotted vertical lines correspond to the estimated theoretical magnitude limits from Eq. (2); the turnovers in the counts given in this figure indicate the detection completeness for these cluster images. Both values are reported in Table 4 for all clusters. The dashed line is the cumulative stars+field galaxies distribution expected to be found in the area of the mosaic.

At the final step, the consistency among the previous three diagrams (Figs. 9, 7 and 8) is used as an a posteriori quality check, as well as as a good empirical tool for possible refinement of SExtractor's input parameters. Usually, when this ideal combination is achieved, objects are detected down to the completeness limit without populating the source lists with unwanted spurious detections.

\subsection{Astrometry}

Most of the non-linear distortion of WFCAM over the entire field is accounted for, by a cubic radial term in the astrometric solution. The CASU pipeline processes the raw images considering the differential field distortion too, giving at the end an astrometric error usually below $50 \operatorname{mas}^{9}$ (for further details refer to CASU website, and Dye et al. 2006). We are able to reach that precision only in relative astrometry, i.e. difference of coordinates in two bands of the same field. After the coadding of all the MEFs into a single mosaic, an additional astrometric and photometric re-calibration check with point-like sources from the 2MASS catalogue is performed. Figure 10 is a visualization of the astrometric precision and accuracy for our entire cluster collection: upper panels show the overall spread of the right ascension and declination differences between the positions of common point-like sources in our catalogs and in 2MASS (left panel) or UCAC2 (right panel). Only sources with a photometric error lower than $0.1 \mathrm{mag}$ were considered. The overall zero point shift is negligible for all our applications, and the rms is consistent with UKIRT-WFCAM standard requirements, being of the order of 100 mas ( $r m s=112$ mas).

\footnotetext{
${ }^{9}$ Milliarcseconds.
} 


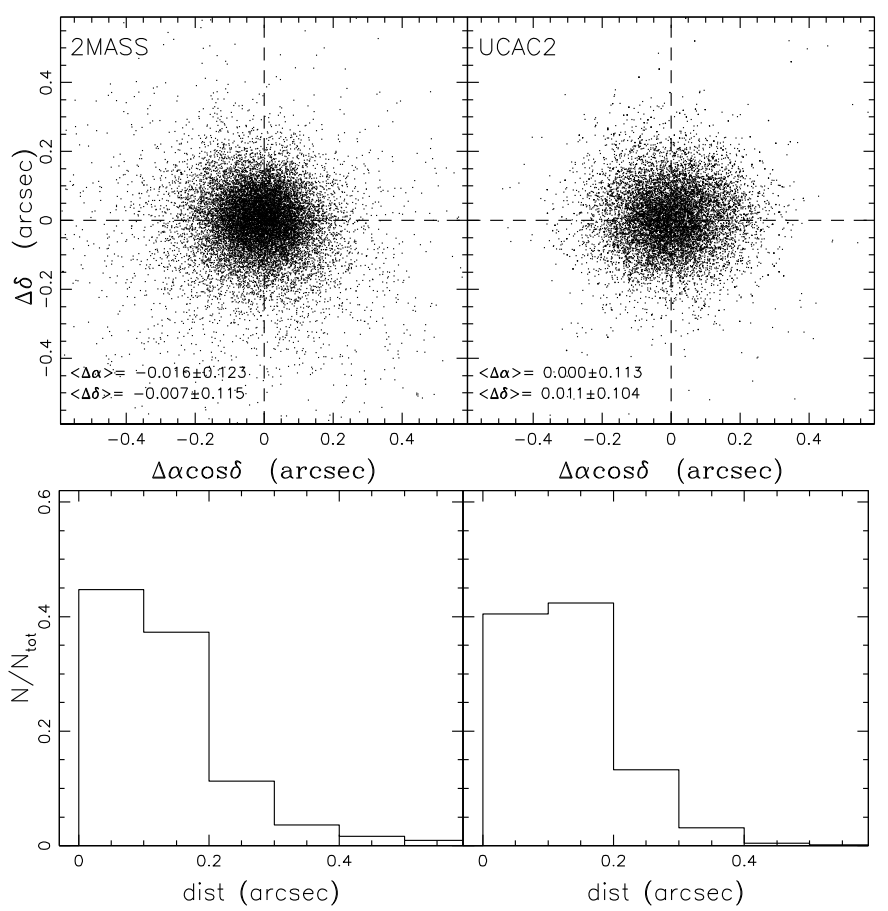

Fig. 10. Astrometry of the WINGS-NIR survey, compared with 2MASS point-source and UCAC2 catalogs (upper panels) and histograms of the distances (bottom panels). The overall precision is of the order of 100 mas and nearly $70 \%$ of the offsets calculated are below one pixel distance. The high precision astrometry of our survey allows safe crossmatching of catalogues extracted from other surveys.

While the original stacks of each tile use a Zenithal Polynomial projection (ZPN) astrometric solution, the final coadded mosaics are expressed in the standard gnomonic tangential projection (TAN). Thanks to the accurate and precise astrometric solutions applied to our mosaics, cross-matched source lists in different bands (optical and $U$ bands included) are very easily obtained, and the fraction of mismatches is negligible.

\subsection{Photometry}

In Table 4 a summary of the properties of the catalogs is given, and the surface brightness limits, calculated in the following way, are reported:

$\mu_{\text {lim }}=\mathrm{ZPT}-2.5 \log _{10}\left[v \sigma_{\mathrm{BG}}\right]+2.5 \log _{10}\left[A^{\prime \prime}\right]$

where $A^{\prime \prime}$ is the pixel area in arcseconds. This relation gives the minimum surface brightness a pixel can have due to the sigma clipping chosen for the specific mosaic. The output of Eq. (4) is obviously changing from cluster to cluster, but it generally settles at $\mu_{J \lim } \approx 22$ and $\mu_{K \lim } \approx 21$.

Photometry at CASU is currently based on 2MASS, via colour equations converting 2MASS magnitudes to the WFCAM photometric system. The most recently released photometric calibrations are given by (see, Hodgkin et al. 2009). Neglecting the interstellar extinction, they are as follows:

$$
\begin{aligned}
J_{\text {WFCAM }} & =J_{2 \text { MASS }}-0.065\left(J_{2 \text { MASS }}-H_{2 \text { MASS }}\right) \\
K_{\text {WFCAM }} & =K_{2 \text { MASS }}+0.010\left(J_{2 \text { MASS }}-K_{2 \text { MASS }}\right) .
\end{aligned}
$$

Due to the improved precision in the fitting algorithm, these equations are different from those given in the early-data-release by Dye et al. (2006). Different tests carried out at CASU suggest that the 2MASS calibration is delivering photometric zero-points

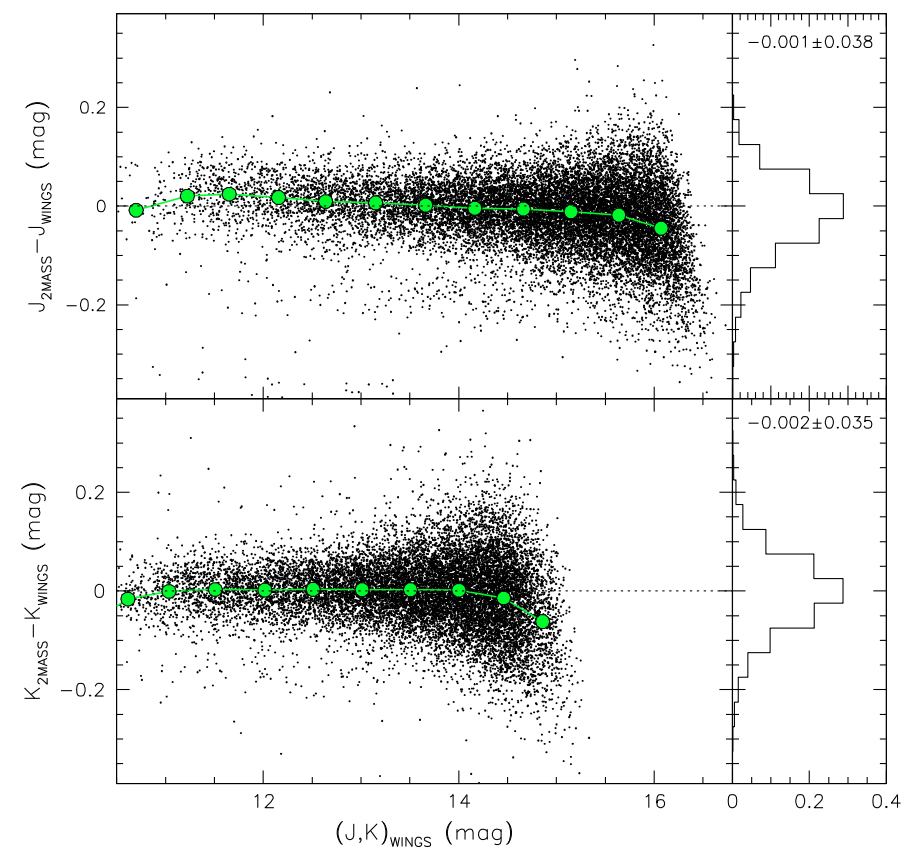

Fig. 11. The 2MASS photometry is converted to the WINGS/WFCAM photometric system with Eqs. (5), (6) and then compared with WINGS magnitudes, for the whole collection of our NIR mosaics, separately in $J$ - (upper panel) and $K$-band (lower panel). For a correct comparison with 2 MASS we use aperture (3". 0 diameter) corrected magnitudes in the $Y$ axis. The absence of a significant zero point shift and the relative tightness of the magnitude sequence demonstrates the quality of the photometry in the WINGS survey.

at the $\pm 2 \%$ level, confirming the excellent results achieved by the 2MASS survey team in ensuring a reliable all-sky accurate calibration. While coadding the single MEFs to obtain the final mosaic, a photometric re-calibration check based on 2MASS catalogs is performed again, to assure a spatially homogeneous zero point throughout the mosaic. Figure 11 shows the difference between 2MASS and WINGS aperture corrected magnitudes of point-like sources (after applying Eqs. (5) and (6), vs. WINGS total magnitudes in the $J$ (upper panel) and $K$ (lower panel) bands, for the complete survey. No significant zero point shift is recovered, confirming the accuracy of the photometric calibration of our WINGS-NIR survey.

Photometric errors assigned by SExtractor are unrealistically small, because they are based only on the photon-noise statistics. The use of the so called confidence-maps (weightmaps for SExtractor users) helps in computing more realistic values of photometric uncertainties. However, we have verified that this latter approach still leads to underestimates of the photometric errors. Therefore, we preferred to use the simulations to recover the effective precision of our measurements. Nevertheless, SExtractor errors will be available in the WINGS website queries forms.

In Fig. 12 we present the global results ( $J$ and $K$ band together) of photometry checks from the simulations. In the top panel the differences between input and output SExtractor magnitudes as measured from the mock images for stars (red starred dots), exponential disks (open magenta circles) and spheroidal $r^{1 / 4}$ (blue filled circles) are plotted for 0.5 mag bins of total magnitude. The agreement is quite good, apart from the constant shift of 0.2 mag for spheroidal galaxies even at bright magnitudes. This effect is well known from the literature (Franceschini et al. 1998) and is also recalled in Paper II. 
Table 4. WINGS-NIR catalogs useful parameters ${ }^{a}$.

\begin{tabular}{|c|c|c|c|c|c|c|c|c|c|c|}
\hline \multirow[t]{2}{*}{ Cluster } & \multirow[t]{2}{*}{ DM } & \multirow{2}{*}{$\begin{array}{c}\text { Band } \\
\text { mag }\end{array}$} & \multirow{2}{*}{$\begin{array}{l}m_{\lim } \\
\mathrm{mag}\end{array}$} & \multirow{2}{*}{$\begin{array}{c}\mu_{\text {thresh }} \\
\text { mag/"/2 }\end{array}$} & \multirow{2}{*}{$\begin{array}{c}\text { Cum } \\
\text { Det }\end{array}$} & \multicolumn{2}{|c|}{ Stars } & \multicolumn{2}{|c|}{ Gals } & \multirow[t]{2}{*}{ Notes } \\
\hline & & & & & & Det & Clas & Det & Clas & \\
\hline A119 & 36.46 & $K$ & 21.2 & 21.02 & 20.0 & 19.8 & 18.2 & 19.3 & 19.0 & \\
\hline A376 & 36.66 & $J$ & 21.5 & 22.11 & 20.2 & 20.4 & 19.0 & 20.0 & 19.6 & 1 \\
\hline \multirow{2}{*}{ A500 } & \multirow{2}{*}{37.40} & $J$ & 21.6 & 21.93 & 21.0 & 20.0 & 18.0 & 19.6 & 19.2 & 1,2 \\
\hline & & $K$ & 20.8 & 21.02 & 19.7 & 19.2 & 17.7 & 18.7 & 18.3 & 2,4 \\
\hline A602 & 37.22 & $K$ & 21.2 & 21.68 & 20.0 & 20.3 & 18.7 & 19.3 & 19.2 & 1,3 \\
\hline \multirow{2}{*}{ A957x } & \multirow{2}{*}{36.43} & $J$ & 21.5 & 22.11 & 20.3 & 20.3 & 18.5 & 19.8 & 19.4 & 2,4 \\
\hline & & $K$ & 21.1 & 21.49 & 20.0 & 20.0 & 18.7 & 19.7 & 19.4 & \\
\hline \multirow{2}{*}{ A970 } & \multirow{2}{*}{37.10} & $J$ & 21.8 & 22.31 & 21.0 & 20.6 & 18.6 & 20.0 & 19.5 & 2 \\
\hline & & $K$ & 20.9 & 21.13 & 19.7 & 19.5 & 17.7 & 18.9 & 18.5 & 1,4 \\
\hline \multirow{2}{*}{ A1069 } & \multirow{2}{*}{37.33} & $J$ & 21.9 & 21.95 & 21.2 & 20.2 & 18.6 & 19.8 & 19.5 & 5,6 \\
\hline & & $K$ & 20.6 & 21.32 & 19.2 & 20.3 & 18.7 & 19.7 & 19.4 & \\
\hline \multirow{2}{*}{ A1291 } & \multirow{2}{*}{36.85} & $J$ & 21.8 & 22.37 & 21.0 & 21.1 & 19.2 & 20.3 & 20.2 & \\
\hline & & $K$ & 20.6 & 21.29 & 19.5 & 19.5 & 18.2 & 19.0 & 18.8 & $1,2,5$ \\
\hline \multirow{2}{*}{ A1631a } & & $J$ & 21.2 & 22.09 & 20.0 & 20.1 & 18.5 & 19.5 & 19.1 & \\
\hline & 36.56 & $K$ & 20.7 & 21.02 & 19.1 & 19.7 & 18.4 & 19.3 & 18.7 & 1,3 \\
\hline & & $J$ & 21.8 & 21.98 & 20.2 & 20.7 & 19.2 & 20.0 & 19.6 & 1,4 \\
\hline A1644 & 36.61 & $K$ & 20.6 & 20.90 & 19.0 & 19.5 & 18.1 & 18.9 & 18.7 & $1,4,6$ \\
\hline & & $J$ & 22.0 & 22.27 & 21.5 & 20.1 & 18.0 & 19.8 & 18.0 & 2,5 \\
\hline A1795 & 37.24 & $K$ & 20.8 & 21.07 & 19.0 & 19.1 & 18.0 & 18.7 & 18.5 & 2 \\
\hline & & $J$ & 21.7 & 22.34 & 21.1 & 21.0 & 19.5 & 20.5 & 20.1 & 6 \\
\hline A1831 & 37.20 & $K$ & 20.5 & 21.19 & 19.3 & 19.3 & 17.5 & 19.0 & 18.6 & 1,6 \\
\hline & & $J$ & 21.9 & 22.63 & 20.6 & 21.0 & 19.5 & 20.3 & 20.0 & 1,4 \\
\hline A1983 & 36.43 & $K$ & 20.2 & 20.74 & 18.9 & 19.0 & 18.0 & 18.5 & 18.5 & 4 \\
\hline A1991 & 37.10 & $J$ & 21.7 & 22.10 & 21.1 & 20.8 & 19.0 & 20.0 & 19.5 & \\
\hline & & $J$ & 21.7 & 22.22 & 21.2 & 20.7 & 19.1 & 20.0 & 19.6 & 2 \\
\hline A2107 & 36.30 & $K$ & 20.8 & 21.08 & 19.2 & 19.2 & 18.0 & 19.3 & 19.1 & 1,4 \\
\hline & & $J$ & 21.7 & 22.29 & 21.2 & 20.2 & 18.7 & 20.0 & 19.7 & \\
\hline A2124 & 37.35 & K & 21.1 & 21.36 & 20.0 & 19.8 & 18.3 & 19.2 & 19.1 & \\
\hline A2149 & 37.43 & $K$ & 20.9 & 21.11 & 20.0 & 19.7 & 18.3 & 19.3 & 19.0 & 6 \\
\hline & & $J$ & 21.7 & 22.14 & 21.0 & 20.8 & 19.1 & 20.1 & 19.8 & \\
\hline A2169 & 37.09 & K & 20.6 & 20.99 & 19.7 & 19.5 & 18.2 & 19.3 & 18.9 & \\
\hline A2382 & 37.21 & K & 20.3 & 20.57 & 19.5 & 19.2 & 17.7 & 18.8 & 18.3 & 2 \\
\hline A2399 & 37.07 & K & 20.8 & 21.04 & 19.6 & 19.5 & 18.2 & 19.2 & 18.6 & 1,4 \\
\hline A2457 & 37.12 & $K$ & 20.9 & 21.14 & 19.8 & 19.7 & 18.0 & 19.3 & 18.7 & 4 \\
\hline A $2572 a$ & 36.25 & K & 21.0 & 21.25 & 19.9 & 19.7 & 18.4 & 19.3 & 19.2 & 2,5 \\
\hline A2589 & 36.31 & K & 21.1 & 21.36 & 20.0 & 19.8 & 18.0 & 19.5 & 18.8 & 2,5 \\
\hline & & $J$ & 21.5 & 21.83 & 20.4 & 20.5 & 19.0 & 19.8 & 19.0 & $1,2,4$ \\
\hline IIZW 108 & 36.70 & K & 21.03 & 21.32 & 19.8 & 19.8 & 18.3 & 19.5 & 19.0 & $1,4,6$ \\
\hline & & $J$ & 21.9 & 22.56 & 20.5 & 21.0 & 19.6 & 20.3 & 20.0 & 1 \\
\hline MKW3s & 36.50 & $K$ & 21.0 & 21.35 & 20.0 & 19.7 & 18.3 & 19.3 & 18.7 & 2,4 \\
\hline & & $J$ & 21.5 & 22.10 & 20.2 & 20.5 & 18.9 & 19.9 & 19.6 & 2 \\
\hline RX102 & 36.6 & $K$ & 21.2 & 21.53 & 20.1 & 19.8 & 18.2 & 19.3 & 19.2 & 2 \\
\hline RX1740 & 36.40 & K & 20.6 & 20.86 & 19.1 & 19.1 & 17.8 & 18.7 & 18.2 & $1,2,4$ \\
\hline 78338 & & $J$ & 21.7 & 22.16 & 20.9 & 20.7 & 19.2 & 20.0 & 19.9 & 2 \\
\hline Z8338 & 36.61 & $K$ & 20.7 & 21.06 & 19.5 & 19.6 & 18.6 & 19.3 & 18.9 & \\
\hline
\end{tabular}

${ }^{a}$ The Cluster column identifies the cluster, the DM column is the cluster distance modulus in magnitudes, the $m_{\text {lim }}$ column is the theoretical magnitude detection limit calculated with Eq. (2), $\mu_{\text {thresh }}$ column is the minimum surface brightness at the detection threshold cut calculate with Eq. (4), the cumulative detection column ("Cum Det") lists the turnover magnitude of the number counts diagram for all the detected sources (see Fig. 9), "Stars" and "Gals" columns represent the average 90\% completeness detection and classification limits for stars and galaxies as deduced from simulations.

Notes legend:

1:= patchy and/or noisy background; $2:=$ strongly variable PSF; $3:=$ many spurious detections; $4:=$ excess of classified stars; $5:=$ deficient in classified stars; $6:=$ exceptionally bright $\operatorname{star}(\mathrm{s})$. 


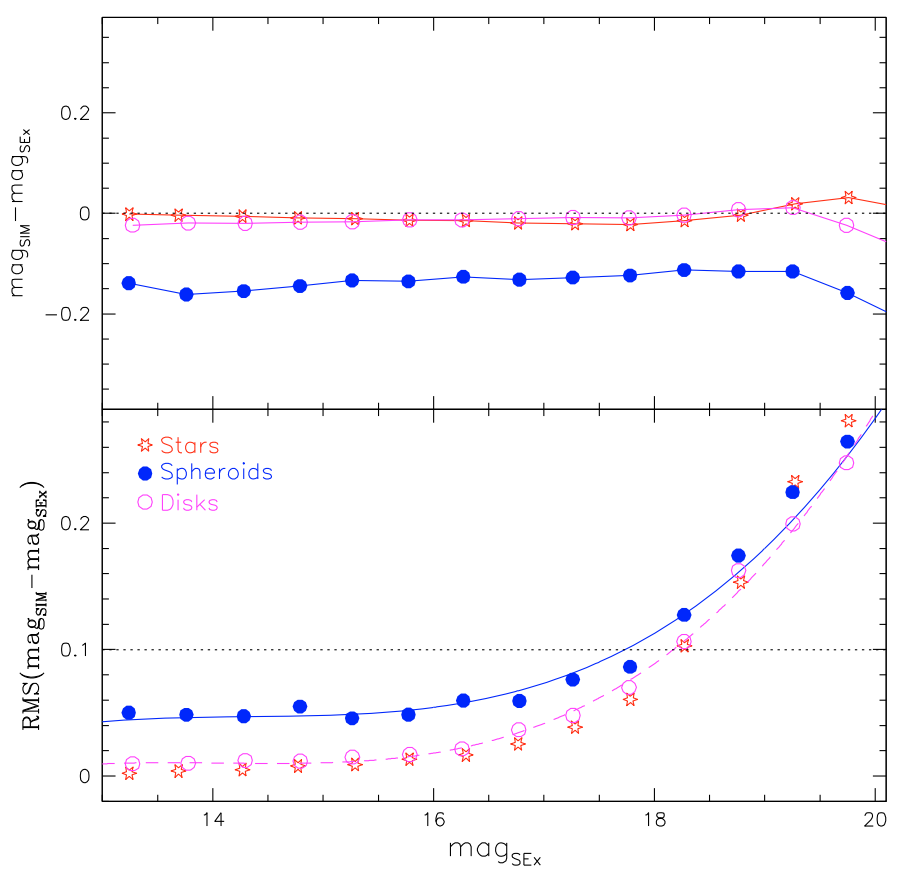

Fig. 12. Global photometric precision and accuracy as obtained from simulations for stars (red starred dots), disks (open magenta circles) and spheroids (full blue circles). Top panel: average differences between the input and SExtractor magnitudes for bins of 0.5 mag of input total magnitude. Bottom panel: rms of the previous differences. While the magnitudes of stars and disks are perfectly recovered, there is a systematic shift of $0.2 \mathrm{mag}$ in the spheroidal $r^{1 / 4}$ magnitudes, due to the loss of light in the wings of simulated galaxies.

It is caused by the loss of light from the wings of profiles of spheroidal $r^{1 / 4}$ galaxies in the background noise. The bottom panel of the same figure presents the overall precision of our photometry (the rms of measurements is plotted for $0.5 \mathrm{mag}$ bins, the symbols are as above), with the corresponding polynomial fit for spheroids (blue full line) and exponential disks (magenta dashed line) to be used as the rms value for all magnitudes of the catalogs:

$$
\begin{aligned}
\mathrm{rms}_{\text {early }}= & -3.675908+0.778114 m_{\text {early }} \\
& -0.054269 m_{\text {early }}^{2}+0.001263 m_{\text {early }}^{3} \\
\mathrm{rms}_{\text {late }}= & -3.765820+0.806960 m_{\text {late }} \\
& -0.057414 m_{\text {late }}^{2}+0.001360 m_{\text {late }}^{3}
\end{aligned}
$$

where $m_{\text {early/late }}$ can be either the total or the aperture magnitude of the corresponding object. Due to the way these errors have been computed, the statistical Poissonian error can be ignored. Moreover Eq. (7) is adequate for the errors associated with the "unknown" objects category, while Eq. (8) can safely be used for point like sources.

Figure 12 shows that the global rms is below 0.1 mag down to $18.3 \mathrm{mag}$ for stars and disks, and almost $18.0 \mathrm{mag}$ for spheroids. It is clear that for $J$-band mosaics this limit can sometimes reach $18.8 \mathrm{mag}$, while for the $K$-band it is found at $\approx 17.5 \mathrm{mag}$, depending on overall quality of the image. Given the overall rates of successful classification deduced from Fig. 7 and Table 3, it is more than conservative to assign those levels of photometric errors beyond $18.0 \mathrm{mag}$ and $17.5 \mathrm{mag}$ for the $J$ and $K$-band, respectively.

As an internal check of photometric accuracy, we show in Fig. 13 two representative examples of total MAG_AUTO colormagnitude diagrams of galaxies from our survey. The tightness

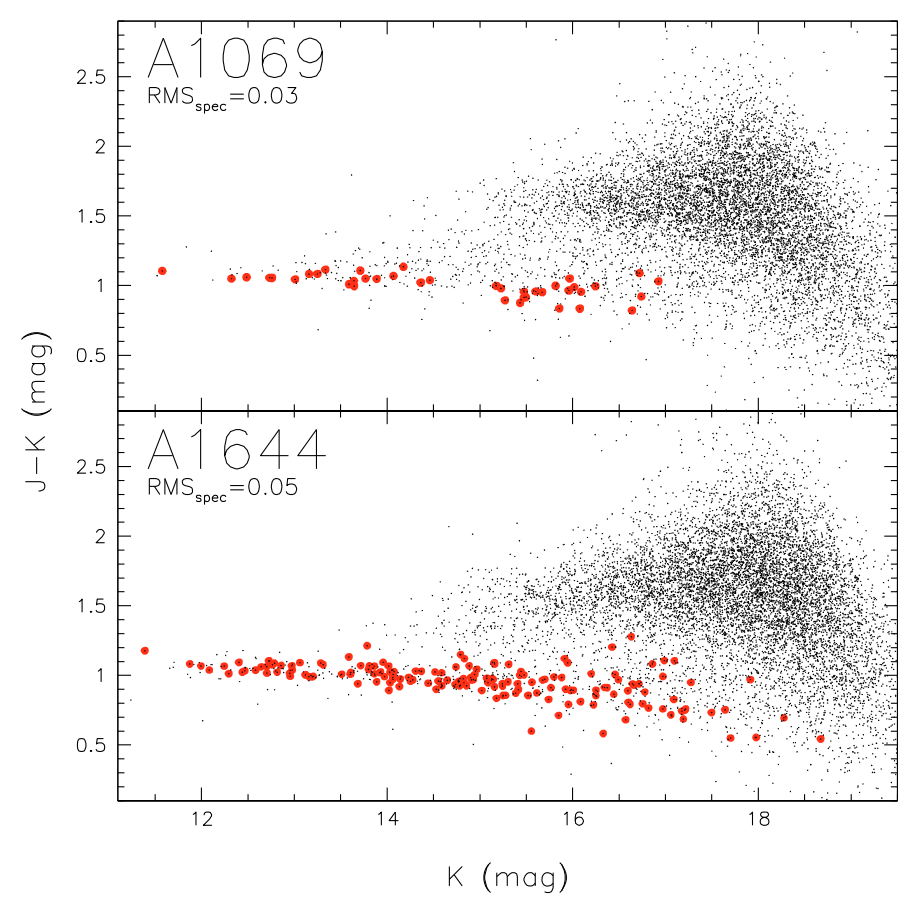

Fig. 13. Color magnitude diagrams of classified galaxies for a selection of WINGS-NIR clusters. The relatively thin red cluster sequences confirm the accuracy of the photometry provided by SExtractor. The large number of detections obtained at fainter magnitudes are the field background galaxies and demonstrate the deepness of our survey. There are also indications of red sequences for the field galaxies at bright magnitudes, indicating the presence of background clusters at higher redshift. The big red full circles are the spectroscopically selected members of the clusters (Cava et al. 2009).

of the red sequences (the rms of the spectroscopically confirmed members of the clusters is $0.03 \mathrm{mag}$ and $0.05 \mathrm{mag}$ ) demonstrates the internal consistency of the WINGS-NIR photometry (these rms values are found also by Eisenhardt et al. 2007).

\section{Summary}

In this paper we have presented the first data release of the WINGS-NIR survey, comprised of $J$ - and $K$-band photometric catalogs for a sub-sample of 28 nearby galaxy clusters belonging to the WINGS optical survey. The detected sources have been classified as stars, galaxies and unknown objects, and for each of them we give positions, geometrical parameters and different kinds of total and aperture magnitudes in both bands (when available).

Due to the variations of the seeing FWHM over the large areas of our mosaics and the poor reliability of SExtractor's CLASS_STAR parameter, we used a specifically designed interactive pipeline to improve star/galaxy classification. The nearinfrared WINGS data consists of nearly one million detected sources, with 150000 and 500000 reliably classified stars and galaxies, respectively.

This unique collection of data turns out to be $90 \%$ complete in detection limits at $J=20.5$ and $K=19.4$, and in classification limits at $J=19.0$ and $K=18.5$ (limits vary from cluster to cluster depending on seeing conditions and total integration time), values to be compared with 2 MASS, $J=15.8$ and $K=14.3$ for point-like sources, UKIDSS Large Area Survey $J=20.0$ and $K=18.4$, and UKIDSS Ultra Deep Survey $J=25.0$ and 
$K=23.0$ (for further details, see Skrutskie et al. 2006; Dye et al. 2006; Lawrence et al. 2007).

Using this new, extensive and comprehensive catalog, we will study several different properties and characteristics of low-redshift cluster galaxies. The near-infrared data presented in this paper will be combined with our optical photometry, morphology and spectroscopy catalogs to systematically study the dependence of these cluster galaxy properties on their stellar mass (since this is traced by the $J$ and $K$ bandpasses). Furthermore, the study of the $J, K$ luminosity functions (known to be a good tracers of the stellar mass function), will allow us to estimate the distribution of the stellar mass-to-light ratio as a function of the cluster-centric distance, for different morphological types. For the galaxies in our sample for which we can derive reliable near-infrared surface photometry and structural parameters (e.g., $R_{\mathrm{e}},\left\langle\mu_{\mathrm{e}}\right\rangle$ ), we will investigate the behaviour of our sample with respect to the various scaling relations (i.e., Fundamental Plane, Kormendy relation). Broad-band spectral energy distributions will be generated for the galaxies in our sample by combining the optical and near-infrared photometry, giving us further information on stellar content and cluster membership. Also, using our cluster sample, we will be looking at what effect the use of a near-infrared total cluster luminosity has on the Fundamental Plane of Galaxy Clusters.

The final version of the complete WINGS survey catalogs will be a comprehensive cross-matched source-list, allowing multiple criteria queries with Web based tools and EuroVO facilities. Through the website the identification of objects which have measurements and calculated quantities from different branches of the survey $(U, B, V, J, K$ photometry, surface photometry, morphology and spectroscopy) will be made easier by means of specific web applets.

Acknowledgements. T. Valentinuzzi acknowledges a post-doc fellowship from the Ministero dell'Istruzione, dell'Università e della Ricerca (Italy). He also thanks for help and useful discussions: Omar Almaini, Sebastien Foucaud, Roberto Caimmi, Stefano Berta, Luca Rizzi, Alessia Moretti, and Stefano Rubele. D. Woods acknowledges the OPTICON travel funding scheme and Australian Gemini Office research funds both which made it possible to obtain these observations at UKIRT. He also thanks his Padova collaborators for their hospitality and generosity during his visit for the team meeting. Marco Riello and Tiziano Valentinuzzi would like to thank Mike Irwin for making the stand-alone versions of the CASU pipeline software available and for helpful discussions and suggestions on the data processing issues related to this work. We also thank the unknown referee for useful suggestions and comments which stimulated discussion and resulted in an improved paper. These observations have been funded by the Optical Infrared Coordination network (OPTICON), a major international collaboration supported by the Research Infrastructures Programme of the European Commissions Sixth Framework Programme. We want to acknowledge the Terapix software group for the immense help bestowed by their software utilities, and CASU for the great job done with pre-reduction of images and in keeping databases. This research has made use of the NASA/IPAC Extragalactic Database (NED) which is operated by the
Jet Propulsion Laboratory, California Institute of Technology, under contract with the National Aeronautics and Space Administration. IRAF (Image Reduction and Analysis Facility) is written and supported by the IRAF programming group at the National Optical Astronomy Observatories (NOAO) in Tucson, Arizona. NOAO is operated by the Association of Universities for Research in Astronomy (AURA), Inc. under cooperative agreement with the National Science Foundation.

\section{References}

Berta, S., Rubele, S., Franceschini, A., et al. 2006, A\&A, 451, 881 Bertin, E., \& Arnouts, S. 1996, A\&AS, 117, 393

Blakeslee, J. P., Franx, M., Postman, M., et al. 2003, ApJ, 596, L143

Casali, M., Adamson, A., Alves de Oliveira, C., et al. 2007, A\&A, 467, 777

Cava, A., Bettoni, D., Poggianti, B. M., et al. 2009, A\&A, 495, 707

De Lucia, G., Poggianti, B. M., Aragón-Salamanca, A., et al. 2007, MNRAS, 374,809

De Lucia, G., Springel, V., White, S. D. M., Croton, D., \& Kauffmann, G. 2006, MNRAS, 366, 499

De Propris, R., Colless, M., Driver, S. P., et al. 2003, MNRAS, 342, 725

Dye, S., Warren, S. J., Hambly, N. C., et al. 2006, MNRAS, 372, 1227

Eisenhardt, P. R., De Propris, R., Gonzalez, A. H., et al. 2007, ApJS, 169, 225

Emerson, J. P., Irwin, M. J., Lewis, J., et al. 2004, in Optimizing Scientific Return for Astronomy through Information Technologies, ed. P. J. Quinn, \& A. Bridger, Proc. SPIE, 5493, 401

Fasano, G., Marmo, C., Varela, J., et al. 2006, A\&A, 445, 805

Foucaud, S., Almaini, O., Smail, I., et al. 2007, MNRAS, 376, L20

Franceschini, A., Silva, L., Fasano, G., et al. 1998, ApJ, 506, 600

Fritz, J., Poggianti, B. M., Cava, A., et al. 2009, in preparation

Fruchter, A. S., \& Hook, R. N. 2002, PASP, 114, 144

Gavazzi, G., Trinchieri, G., \& Boselli, A. 1990, A\&AS, 86, 109

Girardi, L., Groenewegen, M. A. T., Hatziminaoglou, E., \& da Costa, L. 2005, A\&A, 436, 895

Hambly, N. C., Mann, R. G., Bond, I., et al. 2004, in Optimizing Scientific Return for Astronomy through Information Technologies, ed. P. J. Quinn, \& A. Bridger, Proc. SPIE, 5493, 423

Hodgkin, S. T., Irwin, M. J., Hewett, P. C., \& Warren, S. J. 2009, MNRAS, 394, 675

Holden, B. P., Stanford, S. A., Eisenhardt, P., \& Dickinson, M. 2004, AJ, 127, 2484

Holden, B. P., van der Wel, A., Franx, M., et al. 2005, ApJ, 620, L83

Irwin, M., Lewis, J., Riello, M., et al. 2008, in preparation

Irwin, M. J., Lewis, J., Hodgkin, S., et al. 2004, in Optimizing Scientific Return for Astronomy through Information Technologies, ed. P. J. Quinn, \& A. Bridger, Proc. SPIE, 5493, 411

Kauffmann, G., \& Charlot, S. 1998, MNRAS, 297, L23

Kodama, T., \& Bower, R. 2003, MNRAS, 346, 1

Lawrence, A., Warren, S. J., Almaini, O., et al. 2007, MNRAS, 379, 1599

Mei, S., Holden, B. P., Blakeslee, J. P., et al. 2006, ApJ, 644, 759

Pahre, M. A. 1999, ApJS, 124, 127

Poggianti, B. M., Bridges, T. J., Mobasher, B., et al. 2001, ApJ, 562, 689

Riello, M., \& Irwin, M. J. 2008, in The 2007 ESO instrument calibration workshop: proceedings, ESO Astrophys. Symp. (Springer-Verlag), 581

Skrutskie, M. F., Cutri, R. M., Stiening, R., et al. 2006, AJ, 131, 1163

Tokunaga, A. T., Simons, D. A., \& Vacca, W. D. 2002, PASP, 114, 180

Trager, S. C., Faber, S. M., Worthey, G., \& González, J. J. 2000, AJ, 120, 165

van Dokkum, P. G., \& Stanford, S. A. 2003, ApJ, 585, 78

Varela, J., D’Onofrio, M., Marmo, C., et al. 2009, A\&A, 497, 667 